\title{
Quantitative Luminescence Imaging System Description and User's Manual
}

\author{
K. A. Stahl \\ C. R. Batishko
}

June 1988

Prepared for the U.S. Air Force School of Aerospace Medicine, Brooks Air Force Base, Texas Under a Related Services Agreement with the U.S. Department of Energy Contract DE-AC06-76RLO 1830

Pacific Northwest Laboratory Operated for the U.S. Department of Energy by Battelle Memorial Institute 


\section{DISCLAIMER}

This report was prepared as an account of work sponsored by an agency of the United States Government. Neither the United States Covernment nor any agency thereof, nor Battelle Memorial Institute, nor any or their employees, makes any warranty, expressed or implied, or assumes any legal liability or responsibility for the accuracy, completeness, or usefulness of any information, apparatus, product, or process disclosed, or represent ș that its use would not infringe privately owned rights. Reference herein to any specific commercial product, process, or service by trade name, trademark, manufacturer, or otherwise does not necessarily constitute or imply its endorsement, recommendation, or favoring by the United States Government or any agency thereof, or Battelle Memorial institute. The views and opinions of authors expressed herein do not necessarily state or reflect those of the United States Government or any agency thereof, or Battelle Memorial Institute.

\section{PACIFIC NORTHWEST LABORATORY operated by BATTELLE MEMORIAL INSTITUTE for the UNITED STATES DEPARTMENT OF ENERGY under Contract DE-AC06-76RLO 1830}

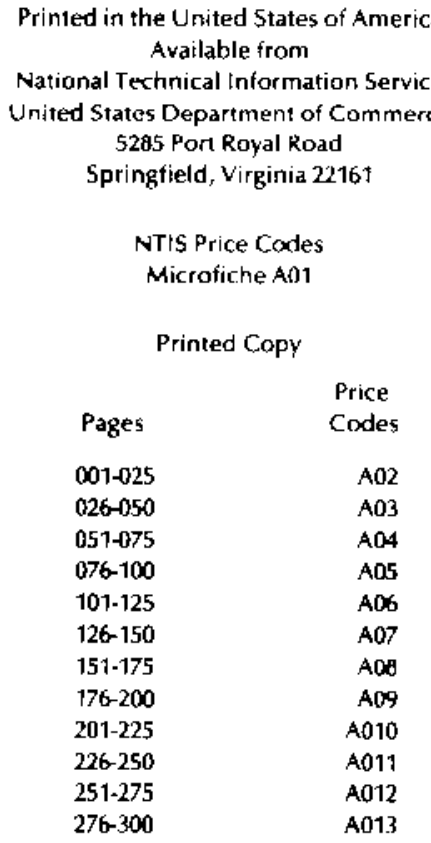


K. A. Stahl

C. R. Batishko

June 1988

Prepared for the U. S. Air Force School of Aerospace Medicine, Brooks Air Force Base, Texas, under a Related Services Agreement with the U. S. Department of Energy Contract DE-ACO6-76RLO 1830

Pacific Northwest Laboratory

Richland, Washington 99352 


\section{ABSTRACT}

A Quantitative Luminescence Imaging System (QLIS) was designed and constructed. The system was developed for use in imaging and quantitative analysis of very low light level chemiluminescent phenomena. The luminescent reactions are imaged via a microchannel plate image intensifier coupled to a newvicon video camera. The video record of the reaction can be stored on video tape or digitally captured by an image processing system which is integral to a host computer controller. Since the particular experimental conditions for which the QLIS was designed necessitate that the chemiluminescent reaction take place in an RF flux within a waveguide, the system includes a coherent fiber optic image transfer system which allows the video hardware to be mounted externally to the RF waveguide.

The QLIS is calibrated with a compact self-luminous light source of known spectral content and radiance. The calibration routine allows quantitative analysis of the chemiluminescent reaction; values for radiance, radiant flux, and photons per second are calculated by the QLIS analysis software.

In addition to the calibration and quantitative analysis software; several programs are provided which provide accessibility to image processor functions. These codes facilitate general image capture and manipulation, image averaging, histograms, and multiple image display.

This document details the QLIS instrumentation, software, and quantitative analysis theory and provides instructions for operation of the system. 


\section{SUMMARY}

Guidelines for use of the Quantitative Luminescence Inaging System (QLIS) have been presented. The hardware was broken down into four major subsystems including the imaging system, calibration source, RF waveguide and structural support, and the computer system. Assembly and adjustment of the various subsystems was explained.

The image processing capability of the QLIS was discussed. The software necessary for control of the image processor was outlined, as was the data analys is software. The procedure required to load the image processor drivers was given.

The requirements for the quantitative analysis of QLIS imagery were addressed. Equations for calculation of radiance, radiant flux, and photons per second were developed. 


\section{CONTENTS}

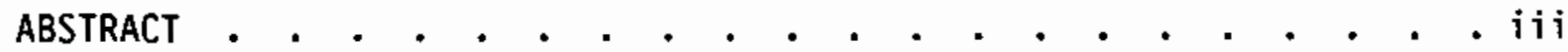

SUMMARY

SYSTEM OVERVIEN.

HARDWARE DESCRIPTION

IMAGING SYSTEM • . . • . . . . . . . . . . . . 3

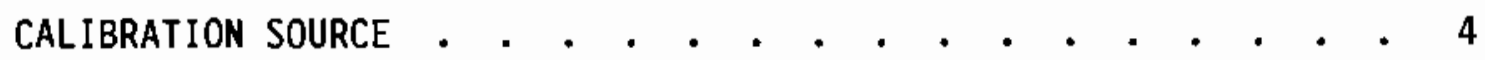

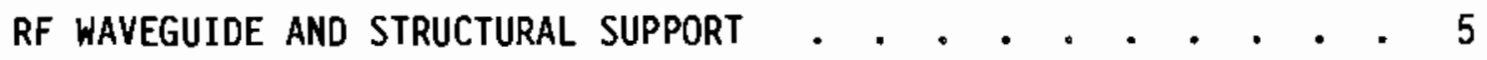

COMPUTER SYSTEM • • • • • • • . • • • • • • • • • • . 7

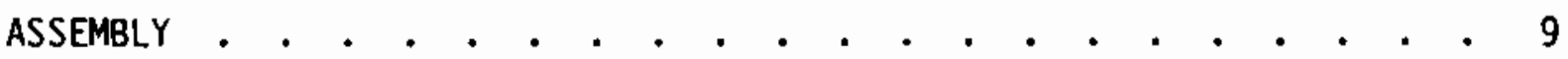

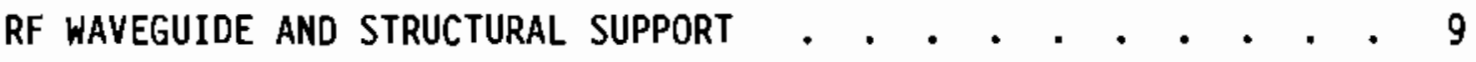

COMPUTER AND IMAGE PROCESSOR . . . • . • . . . . . . . . . 11

IMAGING SYSTEM • • • • • • • . • • • • • • • • . 17

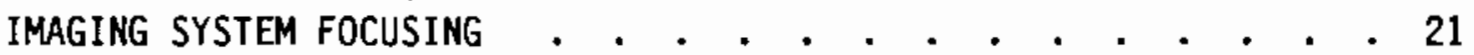

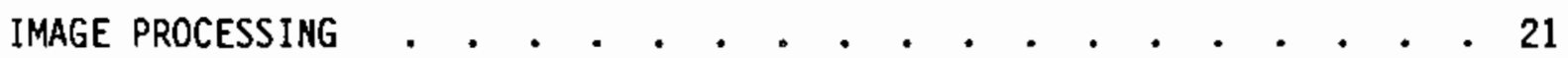

SOFTWARE . . . . . . . . . . . . . . . . . . . 21

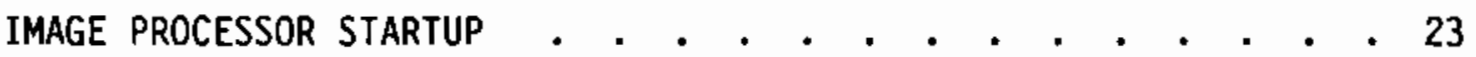

CALIBRATION AND RADIOMETRIC CALCULATIONS

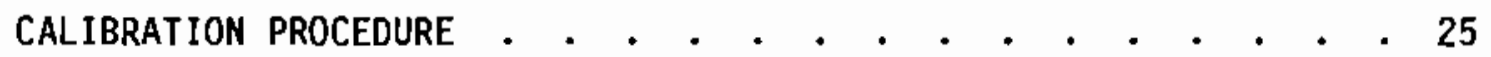

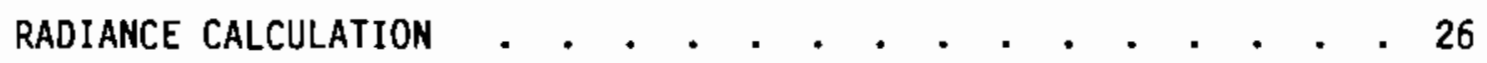

RADIANT FLUX CALCULATION • • • • • • • • • • • • • • • 29

PHOTONS PER SECOND CALCULATION • . . . . . . . . . . . . . 31

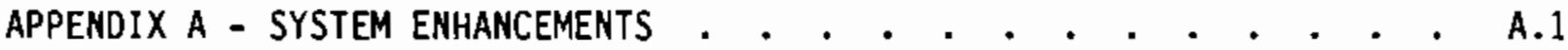

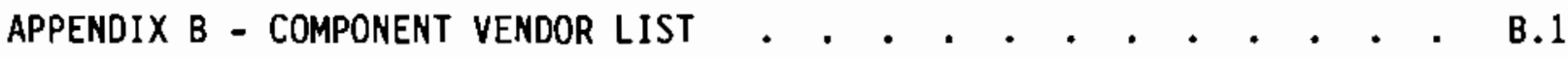

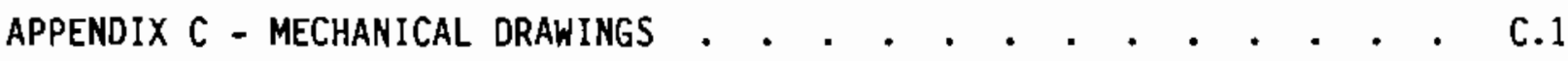


.

-

,

\section{"}




\section{FIGURES}

1 Imaging System Mounted to Haveguide . . . . . . . . . . . 3

2 Complete RF Waveguide, Structural Support, and Imaging System * * 6

3 QLIS Computer System . . . . . . . . . . . . . . . . 7

4 Image Processing Boards in Position in Computer Card Cage . . 10

5 Disassembled Imaging System - • • • • • • • • • • • • 11

6 Disassembled Image Pipe . . . . . . . . . . . . . . . . . 13

7 Typical Index Matching Fluid Application on Small End of Fiber Optic Reducer . . . . . . . . . . . . . . . . . . 14

8 Typical Unevenly Distributed Index Matching at Image Pipe Component Interface . . . . . . - . . . . . . . . 15

g Typical High Quality Image Pipe Component Interface - . • . . 16

10 Graphical Representation of Relationship Between Grey Value, Intensifier Gain, and Scale Factor . • • . • • . . • . . 27

11 Illustration of Spectral-to-Grey Scale Conversion Efficiency: a) Low Efficiency, b) High Efficiency . . . . . . . . . . 29

12 Relationship Between Numerical Aperture and Solid Angle . . . . 30 
, 
QUANTITATIVE LUMINESCENCE IMAGING SYSTEM DESCRIPTION AND USER'S MANUAL

\section{SYSTEM OVERVIEH}

The Quantitative Luminescence Imaging System (QLIS) is a low-light-level imaging and image analysis system developed by the Pacific Northwest Laboratory (PNL) for use in experiments involving very low-light-level chemiluminescence phenomena. The instrumentation represents a prototypical state-of-the-art system for quantitative macroscopic luminescence investigations. 


\section{HARDWARE DESCRIPTION}

The hardware components of the QLIS consist of four major subsystems: the imaging system, the calibration source, the RF waveguide and structural support assembly, and the computer system. Each of these subsystems are in turn composed of a number of individual components which can be discussed in detail. Several enhancements to the QLIS have been added in the period between delivery of the QLIS and the completion of this manual. Since this equipment does not affect the design or basic function of the QLIS, they are discussed briefly in Appendix A. Commercially available components used in the QLIS were purchased from the vendors given in Appendix B.

\section{IMAGING SYSTEM}

The imaging system is made up of a microchannel-plate-based image intensifier optically coupled to a newvicon video camera, along with a coherent fiber bundle and 2:1 reducer which serve to transfer the luminescent image from the RF waveguide interior through the waveguide mesh out to the externally mounted intensifier-newvicon. The system is shown attached to the mounting plate as it would be in operation in Figure 1. The intensifier-newvicon combination

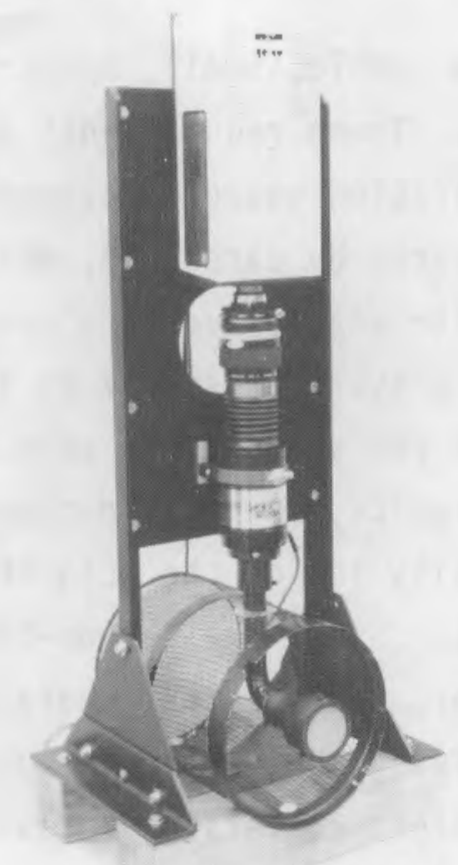

FIGURE 1. Imaging System Mounted to Waveguide 
is available as a standard line product, model \#KS-1380G, from OPELCO, Inc. Note that the two Nikon Nikkor $50 \mathrm{~mm} / 1.2$ transfer lenses which are normally supplied with the KS-1380G have been replaced by a single Nikon Nikkor $35 \mathrm{~mm}$ $f / 1.4$ lens in order to provide a larger field of view.

The coherent fiber optic bundle (image pipe) and 2:1 reducer were purchased from INCOM, Inc. The 2:1 ( 2 inch to 1 inch) reducer is a standard line item, while the 1 inch diameter rigid glass coherent fiber bundle was fabricated on a custom basis. The 2:1 reducer allows a large (1.5 inch diameter) area image to be input to the image intensifier, which has an input window of only 20 millimeters (.8 inches). The reducer is coupled to the image pipe with a viscous index matching fluid to ensure minimal optical loss due to Fresnel reflection at the glass-glass interface. This assembly is held together by a nylon coupler which was designed and fabricated by PNL. It should be mentioned that the two fiber optic components could also be permanently bonded together with UV curing optical adhesive, eliminating the need for the nylon coupler. The output end of the fiber optic assembly is butted against the input window of the image intensifier and held in place by a split ring collar assembly.

\section{CALIBRATION SOURCE}

To calibrate the QLIS, a stable, small, spectrally tailored, low-lightlevel light source is needed. These requirements are met by the carbon-14 activated self-luminous calibration source provided with the QLIS. Since the calibration's source is activated by carbon-14, which has a half life of 5730 years, the source will be quite stable over the useful life of the QLIS. The light source manufacturer, TLS Systems, specifies that the source output will decay by only about 1 percent per year. This source also needs no external power or stabilization electronics, so it is packaged in a very compact diskshaped housing which fits easily inside the QLIS RF cage and mounts easily to the fiber optic reducer input. Since the carbon-14 disintegrations are converted to light via a phosphor, the spectral characteristics of the source can be tuned simply by specifying the particular phosphor to be used when assembling the source. The light conversion mechanism automatically results in low light levels for reasonable amounts (milliCuries) of activation. 
The specific source supplied with the QLIS is a TLS Systems model \#40108-4 with phosphor \#5000 (approximately $520 \mathrm{~nm}$ peak) and a carbon-14 activation of 17.5 millicuries. The source is housed in a sealed package with a plastic window. The source package meets ANSI 542 safety standards. The source is held in a Delrin adapter which allows the source to be coupled to the input of the QLIS for calibration. The adapter also features a sliding shutter which provides a light seal so that the source phosphor is less likely to be exposed to room light. Exposure to room light causes no permanent damage; but after an exposure, the source must be placed in a dark environment for a period of time to allow it to return to its rated intensity.

\section{RF WAVEGUIDE AND STRUCTURAL SUPPORT}

The RF waveguide and structural support system is pictured in Figure 2. The RF cage used by PNL as a guide for design of the QLIS was provided by the sponsor. This waveguide is essentially identical to the actual, powered waveguides to be used in the sponsor's research. The structural support system serves to orient the imaging system with the waveguide so that proper alignment can be easily achieved and maintained. The base of the support system consists of four wooden cradle blocks connected by aluminum angle support members. The two halves of the waveguide are then attached to the cradle block by nylon machine screws which pass through existing holes in the waveguide mesh. This arrangement allows the QLIS to be placed on a lab bench, table, etc., and provides a means to easily slide the two halves of the waveguide apart allowing access to the sample chamber and any other components contained within the waveguide. The cradle blocks have been fabricated with care so that good alignment of the waveguide can be obtained simply by sliding the two halves of the waveguide together until the contact fingers of the two halves are firmly seated.

The remaining support structure is needed to place the relatively bulky imaging system in a properly oriented vertical position above the waveguide. This structure consists of a mounting plate which holds the KS-1380G and mounting plate support members. Vertical slots are machined into the plate at each mounting bolt position so that the entire imaging system (including 


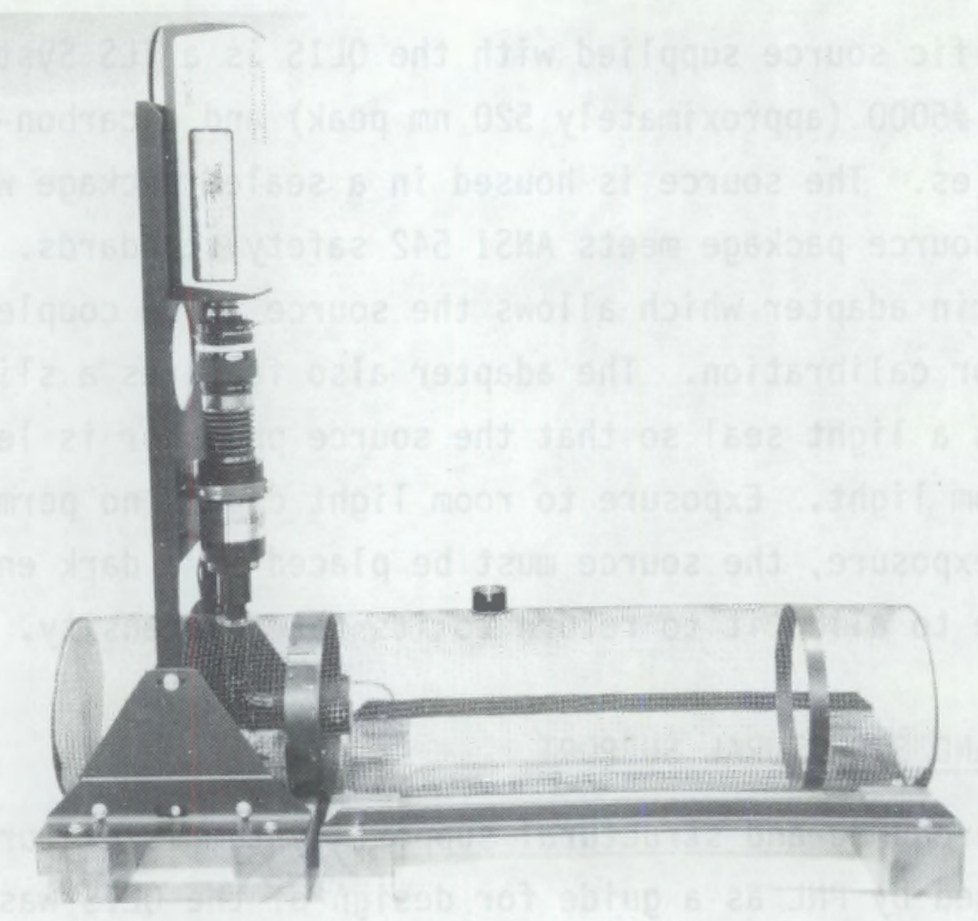

FIGURE 2. Complete RF Waveguide, Structural Support, and Imaging System

the fiber optic assembly) can be moved vertically for alignment with the sample cell inside the waveguide. The slots also facilitate alignment and focusing of the two components making up the KS-1380 during initial assembly. The mounting plate support members attach to vertical plates which are in turn attached to the cradle block assembly. The vertical plates have machined slots which allow for azimuth adjustment of the imaging system. This adjustment provides a means of orienting the input face of the fiber optic reducer to any angle from vertical to approximately \pm 5 degrees. The same set of slots also provides horizontal (longitudinal) movement of the entire imaging assembly.

The structural support system is designed so that no permanent measures such as welds or solders are used in attaching the support system to the waveguide. This design is intended to minimize any difficulties which may arise when transferring the system to a working laboratory waveguide. 


\section{COMPUTER SYSTEM}

The computer system is made up of the host computer, monitor, and image processor and is pictured in Figure 3. The host computer is a Digital Equipment Corporation (DEC) MicroVax II. The computer is fitted with a total of 5 Mbytes of memory, two 71 Mbyte disk drives, and a 95 Mbyte cartridge tape. Other options, such as Ethernet, were specified by the sponsor for experimental and laboratory support and are provided along with the items mentioned above. However, these additional options will not be detailed in this manual since they do not affect operation of the basic QLIS. The monitor is a DEC VT240/241, which serves as a convenient high-resolution RGB monitor for the image processor as well as system monitor for the Microvax II.

The image processor is a board-level system manufactured by Datacube, Inc. The QVG/AFA/SP-423 consists of two mother-daughter board pairs which plug directly into the Microvax II backplane, occupying a total of four quad-height slots. Since the image processor is a board-level unit, a software driver must be employed if the boards' functions are to be called from a high-level language such as FORTRAN. The software drivers (called VIOS) for the boards

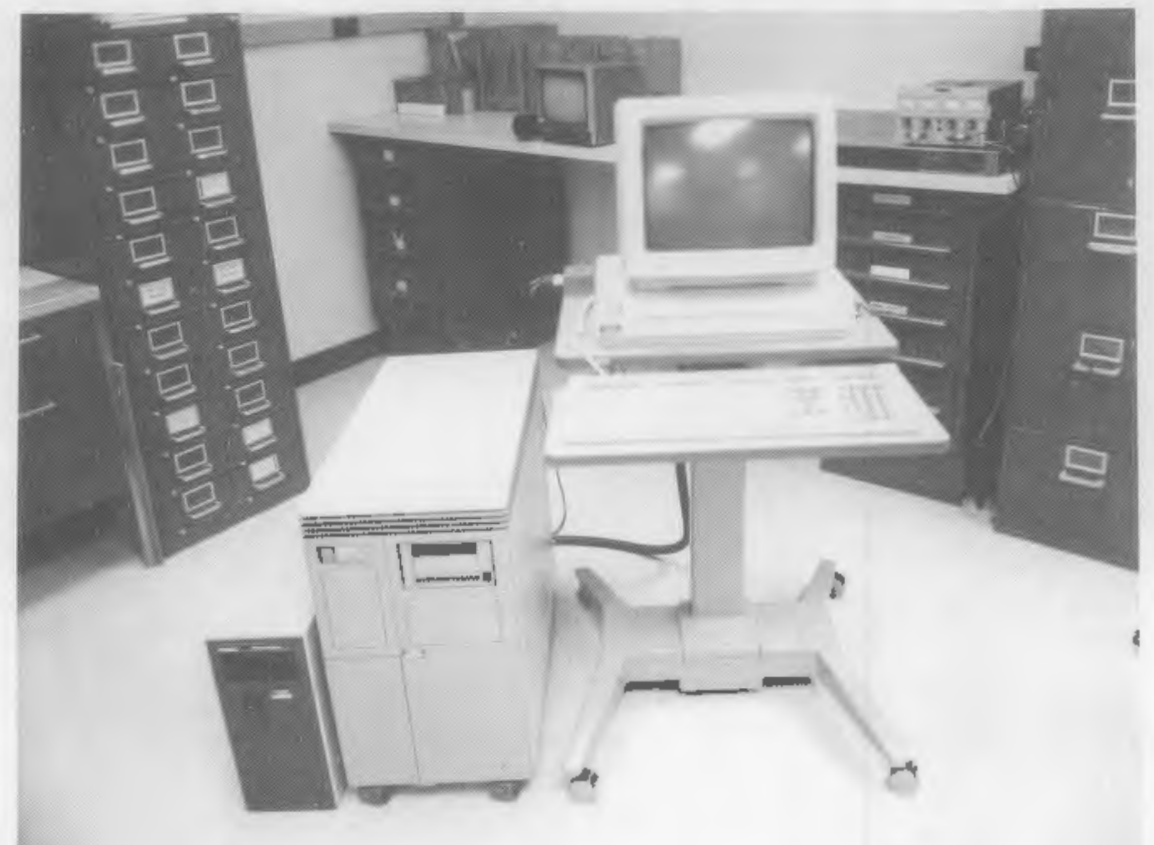

FIGURE 3. QLIS Computer System 
were purchased from Process Software, Inc. Some of the more important functions performed by the boards are real-time digitization and display, multiple look-up table manipulation, and text overlay. The SP board pair also features a pipeline processor-based ALU which allows the implementation of signal processing algorithms at or near real-time. The software developed by PNL, using the VIOS drivers and Datacube boards, is discussed elsewhere in this manual.

An uninterruptable power supply is included for use with the system. The Topaz \#84864-01 is an 800VA unit capable of supplying power for 12 minutes under full load in the event of a power outage. 


\section{$\underline{\text { ASSEMBLY }}$}

\section{RF WAVEGUIDE AND STRUCTURAL SUPPORT}

The assembly of the RF cage and structural support will not be discussed in great detail in this manual. There are no special skills or procedures involved in the assembly of these components, and the drawings included in Appendix $C$ of this manual should give sufficient guidance should disassembly and/or reassembly be required. However, some instruction may be useful for the initial set-up of the QLIS upon receipt from PNL.

The waveguide and structural support hardware were shipped as three preassembled sections. These sections include the two halves of the waveguide, with braces and cradle block supports in place, and the camera mounting plate with support members. The two sections of waveguide need no additional assembly, so all that remains is to attach the mounting plate to the receiving (short) section of the waveguide. The necessary hardware is attached to the mounting plate support members to indicate their intended use. Assembly is as follows: First remove all of the screws, nuts, etc. Next, attach the mounting plate support members to the cradle block braces with a screw. The screw head should end up in the slot which has been machined in the brace. Do not fully tighten these screws yet. Next, hold the mounting plate assembly in a vertical position and use a screw and nut through the slot in the triangleshaped plate to hold the mounting plate in the vertical position. Now fully tighten the lower screws and add a nut to each screw to lock the screw. The waveguide and mounting plate assembly is complete.

\section{COMPUTER AND IMAGE PROCESSOR}

Assembly of the computer and image processor systems is also quite straightforward. The proper assembly of the various DEC components is best explained in the associated DEC manuals and will not be discussed here. In the case of the image processor boards and drivers, the user is again referred to the respective manuals. However, some helpful hints presented here may reduce the amount of detective work needed to accomplish the installation.

Should removal and re-installation of the image processing boards become necessary, the QVG/AFA mother-daughter board pair must be installed closer to 
the computer's CPU than the QVG/SP board pair. The image processing boards are pictured in Figure 4, partially removed from the card cage in the Microvax II. Also, the mother-daughter board pair configuration of the image processor results in empty backplane slots in the computer where the daughter boards takes up slot space but do not plug into the backplane. Therefore, if interrupt capability is desired, a DEC Grant continuity card must be placed in the empty slots. The QLIS has these cards in place. Notice that the continuity cards are not visible with the image processing boards in place; they have been shortened due to space limitations.

The image processor has four co-axial cable video connections. These connections are made with standard BNC connectors at a bulkhead fixture at the rear of the Microvax II. The bulkhead fixture is labeled and color-coded to indicate the proper connections. A separate cable is required for each of the Red, Green, and Blue video signal outputs. These cables go from the appropriate $R, G$, and $B$ bulkhead connectors to the small light blue switch box used to select which signals (image processor RGB or Microvax II RGB) are to be routed to the monitor. Also, one cable is needed from the VIDEO connector on the KS-1380G to the appropriate bulkhead connector (Input \#1 is standard, though \#2 or \#3 can be used provided software changes are made).

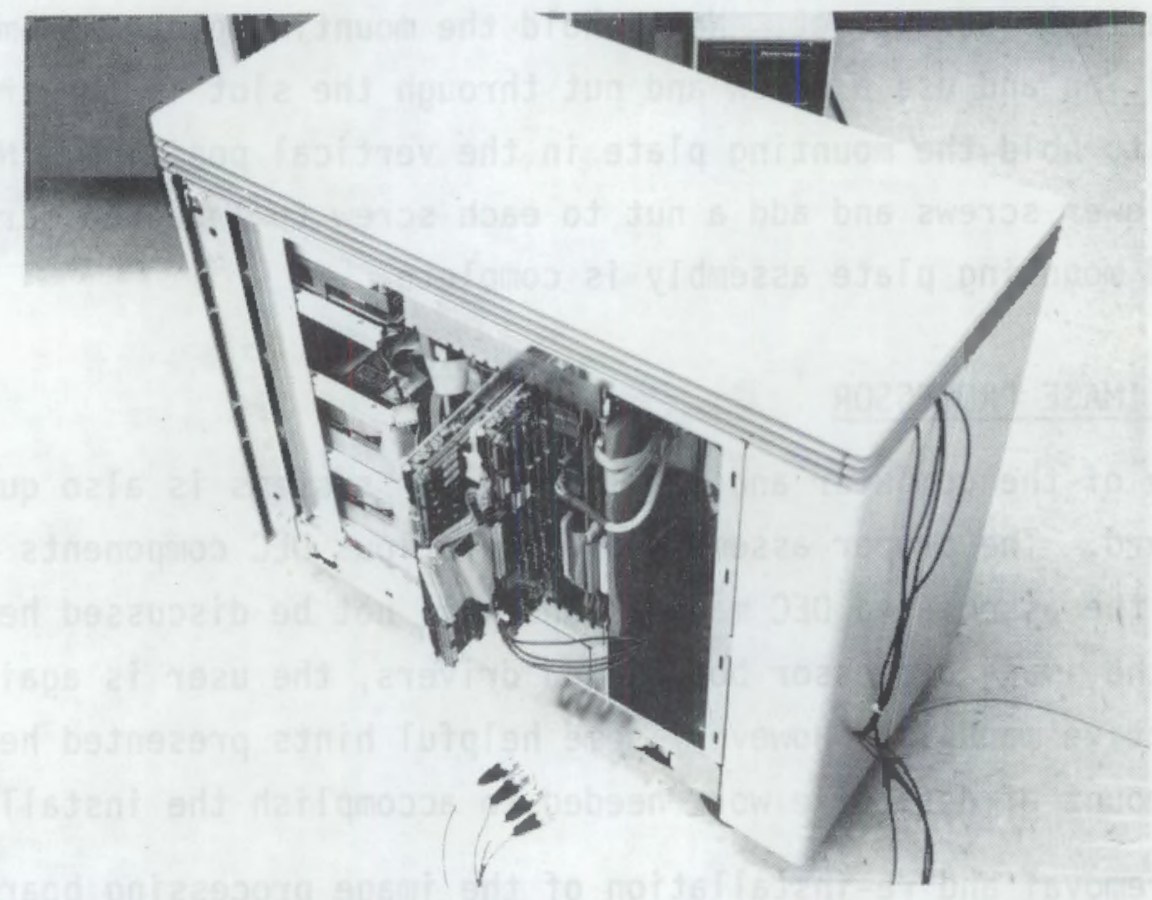

FIGURE 4. Image Processing Boards in Position in Computer Card Cage 
The signals at the bulkhead connections are carried to and from the image processing boards by co-axial cable and standard AMP connectors.

\section{IMAGING SYSTEM}

Assembly of the imaging system involves a number of steps which should be carried out in the order given. In conjunction with these instructions, please refer to the KS-1380G manual provided by the manufacturer, keeping in mind that the transfer lens system has been modified, as mentioned in the HARDWARE DESCRIPTION portion of this manual. The disassembled imaging system is pictured in Figure 5. It is advisable to read and understand all of the following assembly steps before beginning.

1) After assembling the imaging system support structure, the first step should be to attach the newvicon video camera portion of the KS-1380G to the mounting plate. Use two $1 / 4-20$ screws and washers through the vertical slots in the plate and into the tapped holes in the underside of the video camera housing. Leave these bolts a bit slack to allow the camera to be slid up during focusing. During this operation, the protective cap in the lens mount should be left in place as exposure of

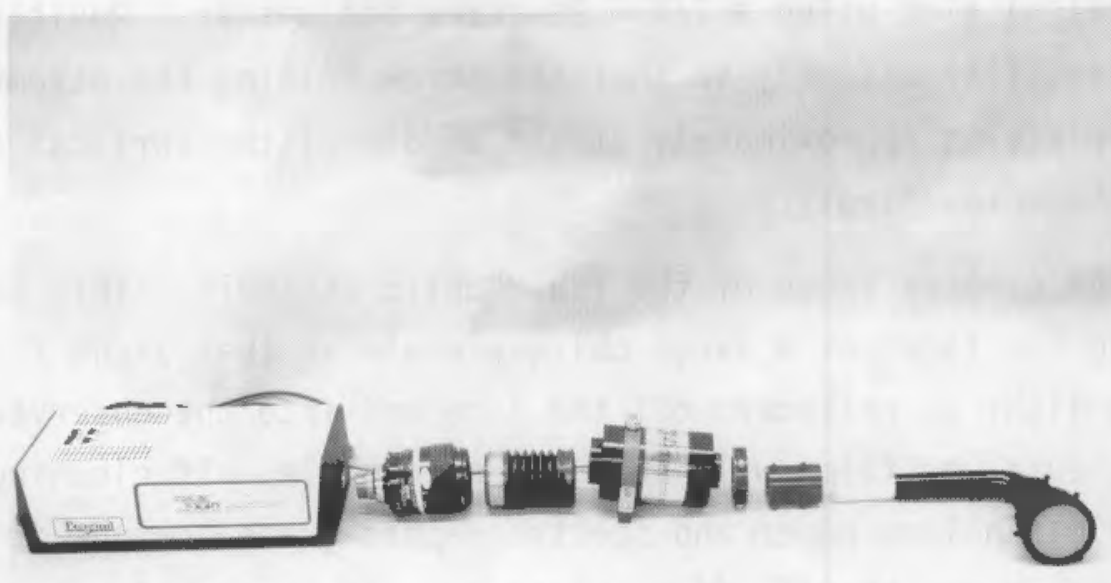

FIGURE 5. Disassembled Imaging System 
the internal newvicon tube to bright light (such as direct room light) may shorten its life.

2) Remove the protective caps from the lens and newvicon and screw-in the C-mounted $35 \mathrm{~mm}$ f1.4 lens and brass extender until firmly seated.

3) With protective caps in place to avoid exposure to light, place the image intensifier tube in the mounting assembly (see drawings in Appendix C) so that the clamping strap is positioned just above the large silver and black label on the intensifier housing, nearest the phosphor screen end, and tighten securely. (This step should not need to be executed for initial set-up; the image intensifier was shipped already strapped into the mount.) To identify which end is the phosphor screen end and which is the input photocathode end, look for the four tube housing screws and the power cable. The screws and power cable are at the photocathode end; therefore, the end which has neither of these features is the phosphor screen end. NOTE: EXPOSURE OF THE PHOTOCATHODE TO BRIGHT LIGHT (GREATER THAN DIMLY LIT ROOM LIGHT) WILL SHORTEN TUBE LIFE OR COMPLETELY DESTROY THE TUBE.

4) Attach the image intensifier tube assembly to the mounting plate at the lower vertical slot using a $1 / 4-20$ screw and washer. Position the image intensifier assembly so that the screw holding the assembly to the mounting plate is approximately at the middle of the vertical slot and tighten the screw firmly.

5) Inspect the exposed faces of the fiber optic assembly. This can be done by viewing the faces at a large oblique angle so that light from an overhead room light is reflected off the face and into the observer's eye. Any dirt, dust, or films will be quite noticeable. If cleaning is necessary, use clean lens paper and spectrographic-grade acetone or ethyl alcohol to remove the offending substance. If the problem is a particulate, such as dust, a jet of clean dry air is often sufficient to remove it and should be tried first. WHEN HANDLING THE FIBER OPTIC COMPONENTS, REMEMBER THAT THEY ARE FRAGILE; THE POLISHED END FACES ARE PARTICULARLY SUSCEPTIBLE TO SCRATCHES AND CHIPS. 
6) If the fiber optics are satisfactorily assembled, skip to step 10. If the assembly is broken down into its individual components, as pictured in Figure 6 , begin by inspecting and cleaning all faces of the fiber optic components. Also, wipe out the inside of the nylon coupler in order to remove excess index matching fluid.

7) The procedure described in steps 7 - 9 will probably require some practice to master, but fortunately it should not need to be carried out very often. Place the reducer on a soft, clean surface such as a piece of velvet or felt, with the large end down. Place several drops of index matching fluid (index between 1.6 and 1.8, such as Cargille 1.638) on the center of the small face of the reducer, forming a "puddle" of fluid as shown in Figure 7 . The index matching fluid is not absolutely critical, but omission will result in lower optical efficiency of hte fiber optic assembly. Place the black nylon coupler over the reducer, being careful to avoid bumping the reducer since a jolt will displace the puddle of index matching fluid. Temporarily tighten the three nylon set screws on the coupler at the large end. Do not overtighten the set screws since the nylon threads are easily stripped.

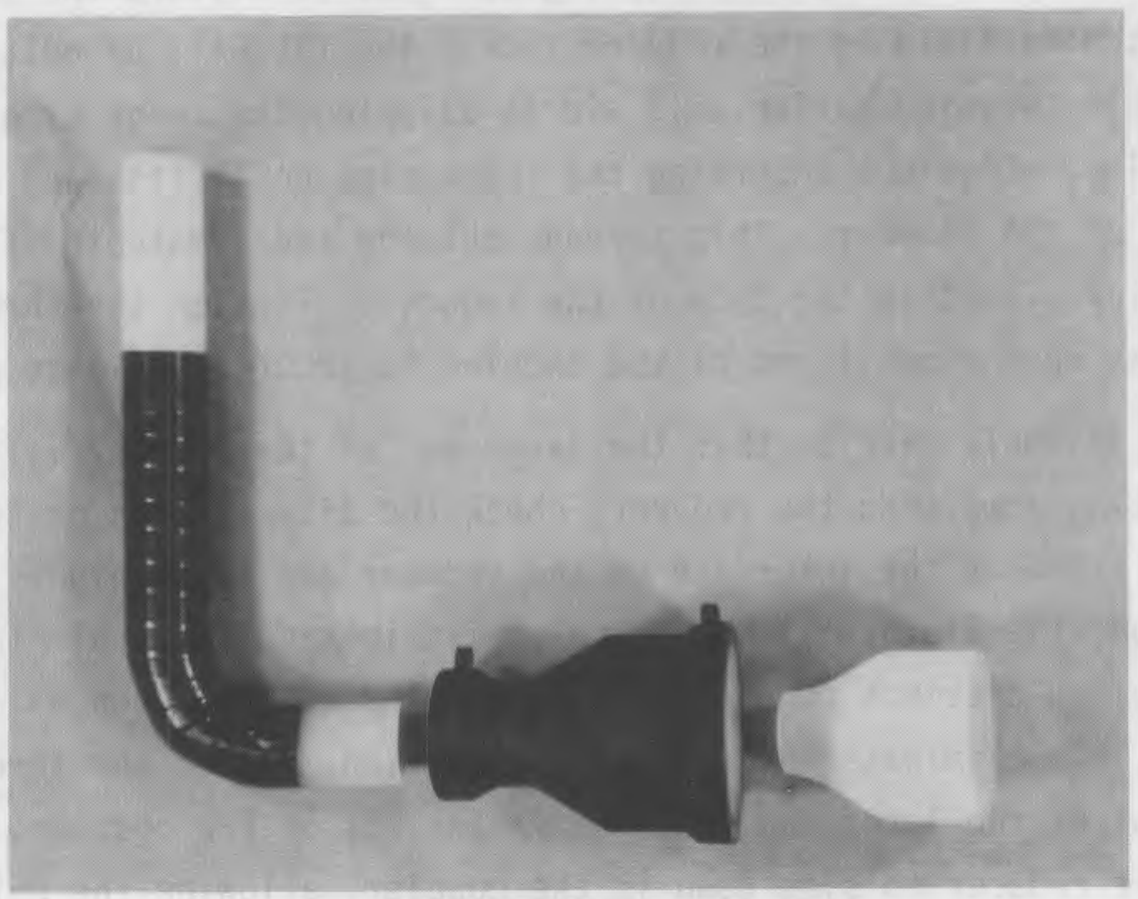

FIGURE 6. Disassembled Image Pipe 


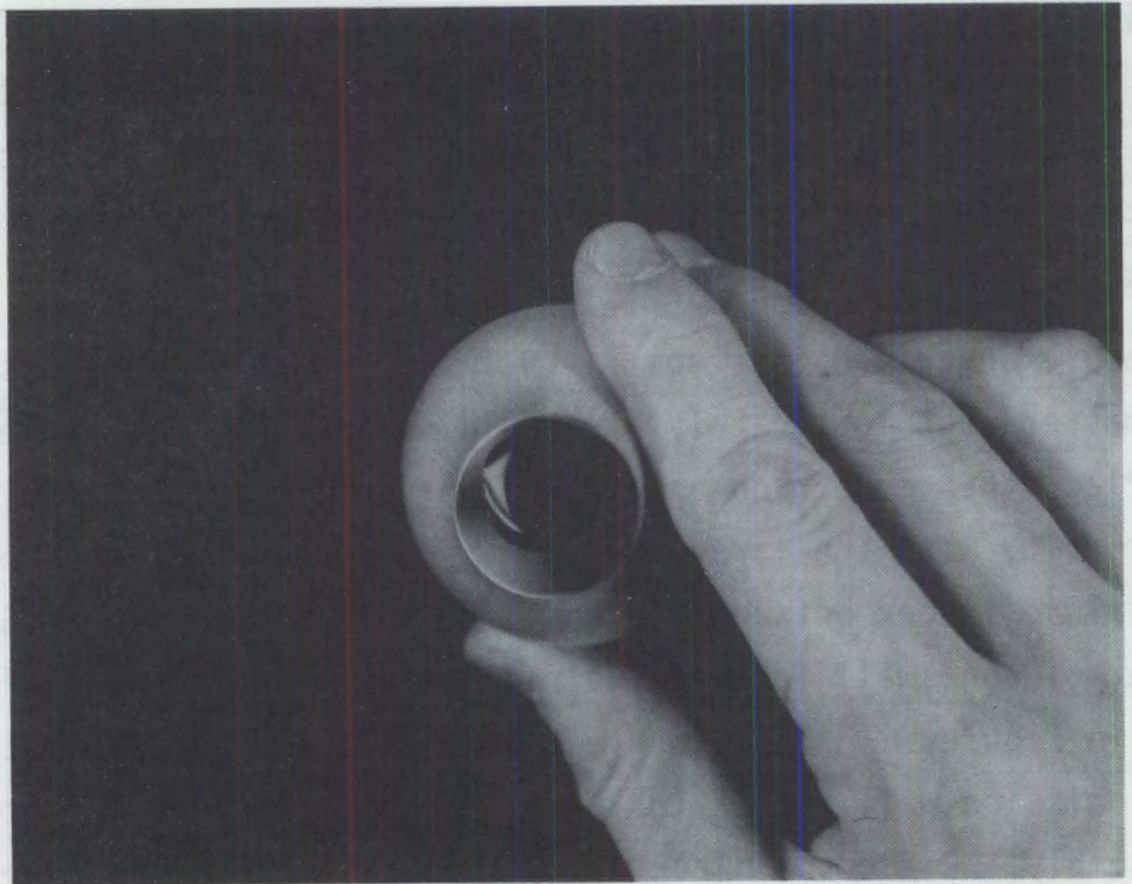

FIGURE 7. Typical Index Matching Fluid Application on Small End of Fiber Optic Reducer

8) Insert the short end of the bent fiber optic image pipe into the small open end of the coupler, still taking care not to disturb the puddle of index matching fluid on the reducer face. The fit will be quite snug, so a gentle twisting motion will aid in slipping the image pipe fully into the coupler. Continue inserting the image pipe until its face meets the face of the reducer. This spreads out the index matching fluid which was earlier placed in the face of the reducer. Tighten the three nylon set screws on the small end of the coupler to secure the image pipe.

9) Turn the assembly over so that the large end of the reducer is facing up. Looking down into the reducer, check the distribution of the index matching fluid at the interface of the reducer and image pipe. In all likelihood, the fluid will be unevenly distributed due to air in the interface, so distinct puddle edges or bubbles will be seen as shown in Figure 8. To eliminate the uneven distribution, loosen the three nylon set screws at the large end (the end facing up) of the coupler. This allows the reducer to slip down in the coupler, allowing the weight of the reducer to aid in squeezing out the excess air in the interface. If 


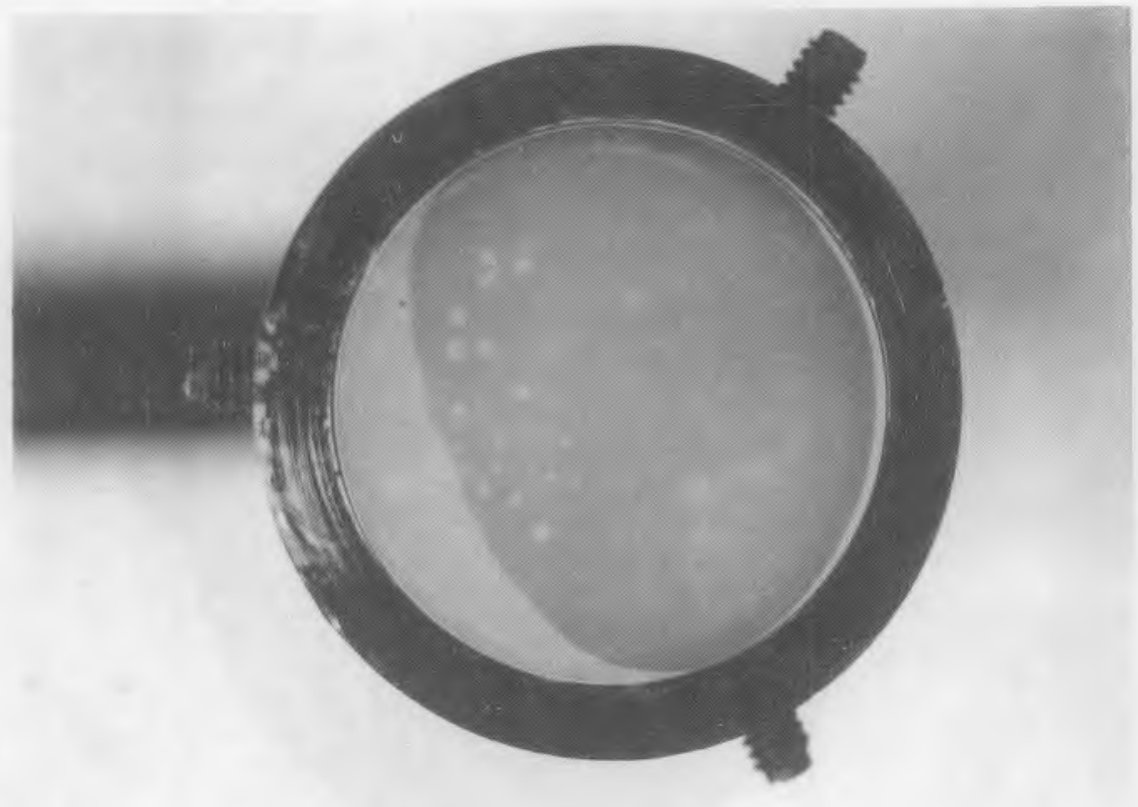

FIGURE 8. Typical Unevenly Distributed Index Matching at Image Pipe Component Interface

all of the air is still not out of the interface, firmly grip the nylon coupler in one hand and with the other hand, turn the image pipe slightly (it will turn stiffly even though the set screws have been tightened). Continue this adjustment until a satisfactory interface is achieved. A good interface is shown in Figure 9. Remember that it is important to ensure that the large face of the reducer is flush with the rim of the nylon coupler when the assembly is completed. When a satisfactory interface is achieved, proceed to tighten the three set screws at the large end of the coupler. Do not tighten one screw completely as this will ruin the airtight seal at the interface. Rather, tighten each screw a little bit at a time. If a turn of a particular screw lets some air into the interface, simply loosen that screw until the interface seals again. There is no need to completely tighten all three screws; one firmly secured screw is normally sufficient.

10) THE EDGES OF THE FACES OF THE IMAGE PIPE ARE QUITE FRAGILE AND WILL SPALL AWAY VERY EASILY. BE VERY CAREFUL WHILE PERFORMING THIS STEP. RemOVe 


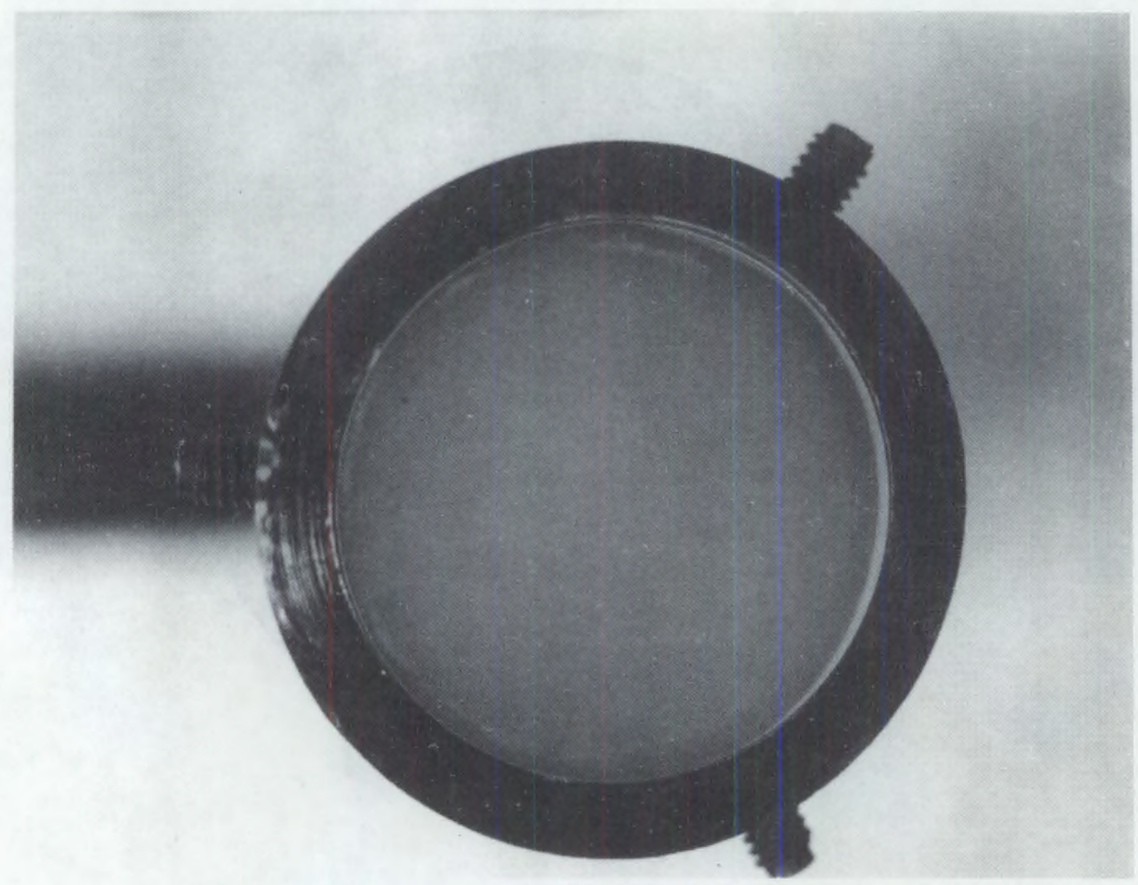

FIGURE 9. Typical High Quality Image Pipe Component Interface

the protective caps from the image intensifier. Working from the open end of the receiver half of the waveguide, carefully run the image pipe end of the fiber optic assembly up through the hole in the waveguide. Continue by slowly inserting the end of the image pipe up into the split ring collar assembly, and on into the image intensifier housing. When sliding the image pipe into the collar assembly, take great care to keep the image pipe in a vertical orientation; otherwise, the image pipe will bind against the collar, chipping the face of the image pipe. Continue inserting the assembly until the end of the image pipe meets the input window of the image intensifier. This will occur just as the black coating on the image pipe meets the rim of the split ring collar assembly. When the image pipe is fully inserted, while looking into the large end of the reducer, the flange around the image intensifier input window will be dimly visible but in good focus. Check to see that the image pipe is arranged so that its optical axis is parallel to the long axis of the waveguide and tighten the six nylon set screws on the split ring collar assembly. The fiber optic assembly is now coupled to the rest of the imaging system. 
11) Loosen the mounting screw which is now holding the image intensifier and fiber optic assembly to the mounting plate. Slide the assembly up or down as needed to position the input to the reducer at the desired vertical height within the waveguide and securely re-tighten the mounting screw. The assembly tends to pivot about the point where it is attached to the mounting plate, so care should be taken to keep the assembly vertical during tightening.

12) If the face of the large fiber optic reducer is not vertical, loosen the screws which hold the mounting plate support members to the waveguide assembly. This will allow the mounting plate to be tilted through approximately \pm 5 degrees from vertical. Tilt the plate as necessary to bring the reducer to vertical. Re-tighten the screws.

\section{IMAGING SYSTEM FOCUSING}

13) The imaging system is now ready to be focused. Turn on the newvicon camera and connect the video output of the camera to the image processor. Select the continuous digitize mode via one of the image processor software routines such as program DISPLAY. Place the video monitor in a position which will facilitate viewing the image while the focusing procedure is carried out. Make sure that the aperture of the $35 \mathrm{~mm}$ lens is open fully $(f / 1.4)$. Dim the room lights, or turn them off completely since the glow of the CRT will probably provide sufficient light for the focusing procedure.

14) NEVER TURN ON THE POWER SUPPLY TO THE IMAGE INTENSIFIER UNLESS YOU ARE MONITORING ITS OUTPUT, in order to avoid inadvertent over-exposure. Connect the cable from the image intensifier to the intensifier power supply. Switch on the intensifier power supply. After approximately 10 seconds, the roughly circular image of the glowing phosphor screen of the image intensifier will appear on the monitor. It will probably be rather badly out of focus. If no image is evident on the monitor, first check that the newvicon is operating and that all cables are properly connected. If the hardware is in order, increase the gain at the image intensifier power supply until an image appears. If the image flashes, immediately decrease the gain until a stable image is achieved. 
15) While supporting the newvicon camera with one hand, loosen the two screws which hold the newvicon camera to the mounting plate. Move the camera up or down until most of the circular image is displayed on the monitor. As the camera is moved, the magnification and centering of the blurry circular image will change. When the displayed image is centered to "eye-ball" accuracy and has sufficient magnification that the circle slightly exceeds the top and bottom boundaries of the video screen, tighten the two mounting screws. Now adjust the focus on the $35 \mathrm{~mm}$ lens for the best image. While focusing, work toward making the random "salt and pepper" noise in the image come into sharp focus. After focusing, repositioning of the newvicon camera may again be necessary in order to get the majority of the circular image onto the monitor. Repeat this step until the image focus and size is satisfactory.

16) Now the focused image must be accurately centered. That is, the newvicon must be tilted to the left or right so that the image of the circular field of view is centered on the monitor screen. Again loosen the two screws which hold the newricon to the mounting plate. Tilt the camera so that a focused and centered image is found on the monitor. This procedure can be carried out quite well using only "eye-balling" to judge when the image is centered. However, since a well-centered image is important, it is advised that one of the software routines such as HISTOGRM or ANALYZE be used to help in centering. Both of these programs feature a selectable "area of interest" which is specified by the operator and subsequently outlined in white on the monitor screen. To use this feature for centering, when the computer prompts for an $X$ and $Y$ coordinate and radius for the area of interest, enter $364,242,242$, which is a centered circle of maximum radius. If the white circle placed on the screen by the computer is not centered on the image, the camera tilt must be readjusted, and the program must again be used to check the 
centering.(a) If the image can be centered with respect to the horizontal but not the vertical, it will be necessary to place a bit of shim material between the intensifier mount and the mounting plate. This is accomplished by loosening the mounting screw with one hand while supporting the intensifier-image pipe assembly with the other. Loosen the intensifier mount just enough to allow the shim to be slid between it and the mounting plate, then re-tighten the intensifier mount screw. As the screw is re-tightened, slide the intensifier assembly as necessary to regain focus and horizontal centering. A well-centered image should be achievable without an excessive number of trials. As with the fiber optic assembly, it is fortunate that this alignment need not be done very often.

17) Return the gain on the image intensifier power supply to zero and switch off the power. Turn the room lights back on. Compress the black rubber bellows and place it between the $35 \mathrm{~mm}$ lens and the upper end of the image intensifier housing, with the threaded end of the bellows facing up. The intensifier end of the bellows simply slips onto the intensifier housing while the lens end screws into the accessory mounting threads on the $35 \mathrm{~mm}$ lens. The imaging system is now fully assembled.

(a) NOTE: The routine of re-selecting the area of interest from the computer program each time a new camera orientation is completed may be eliminated using the following "low-tech" method. With the white circle displayed indicating the area of interest, place a piece of paper against the screen and trace the circle with a pen or pencil. Cut the circle out of the paper and then tape the paper onto the screen with the cut-out circular area of the paper corresponding to the circular area of interest outlined on the screen. Hence, the image processor can be placed in constant digitize mode and the inconvenience of re-selecting the area of interest is no longer required. 



\section{IMAGE PROCESSING}

For a complete description of the image processor, the manuals for the Datacube hardware and Process Software VIOS code should be consulted. In general, the Datacube image processing boards perform all of the basic image processing functions. The boards are accessed and controlled by assemblylevel commands which address various registers on the boards. The VIOS software amounts to a set of drivers which act as an interface between the boardlevel register manipulation and calls from a high-level language such as FORTRAN. Advanced image processing algorithms, such as histogram, transforms, etc., are written by the user in a high-level language using the VIOS calls to act as tools for frame grabbing, look-up table manipulation, text overlay, pixel value read and write, board selection, frame storage and retrieval, and so on. In addition, many real-time ( $30 \mathrm{~Hz})$ algorithms can be implemented using "state tables" written with the VIOS software and utilizing the signal processor part of the image processor. Examples of these types of algorithms include frame summation, frame differencing, and various transforms.

\section{SOFTWARE}

The FORTRAN code provided by PNL consists of a number of individual programs which perform certain algorithms or sets of algorithms. Since new or modified algorithms will doubtless be desired as the QLIS is used, the code provided by PNL should give good examples of how to use the VIOS software and Datacube hardware. The programmer planning to write new algorithms will need to invest some amount of time in becoming familiar with the programming methods, but after becoming acquainted, should find the going about as easy as that for straight programming.

The software provided by PNL includes programs DISPLAY, HISTOGRM, FOURFRAM, FASTAVG, SLOWAVG, ANALYZE, ANALFILE, and CALIBRAT; all are written in FORTRAN. Also, a FORTRAN program called QVTEST as well as several example state tables are provided by Datacube and give additional examples of how to use the image processor. Any of the above programs may be edited to increase their utility or modify their function; the user who is familiar with FORTRAN is invited to do so. 
Program DISPLAY is a menu driven set of algorithms used for selecting an image to be displayed and manipulating the appearance of that image. Functions include frame grab, continuous digitize, restore frame from mass storage, reduce greyscale divisions, negative colorization, interactive colorization, colorization from stored Look-Up Tables (LUTS), iso-contouring, zoom and pan, text on/off, reset LUTs, save image to mass storage, and reset all boards. In many cases, each of these general headings includes a number of subalgorithms, and many choices are presented to the operator throughout the program to allow good flexibility. The algorithms provided by DISPLAY may also be applied to an existing image provided by a previously run program.

The histogram program, HISTOGRM, works on the image presently displayed, a frame grabbed on command, or a previously stored image. The operator may choose between using the entire image or any smaller circular area of interest selected. After computing the histogram, the program can stretch the histogram to maximize the contrast of the image. Numerous options are given to the operator throughout the program.

Also provided is a program which enables the operator to display four inages simultaneously by shrinking each image down to one-quarter of its original size. Each of the four images can be grabbed on conmand from real-time video or pulled up from mass storage. If the resulting quad display is then stored on disk, it can be brought up on a subsequent run of FOURFRAM, resulting in four $1 / 16$-scale images. In this way, a 16 or even a 64 frame display can be built to show the time evolution of a process, image differences, image catalog, and so on.

FASTAVG is a program which performs real-time summation of the incoming video. The user may choose from 2 to 128 frames to be surmed. When the summation is complete, the data is scaled by the number frames summed so that the data is displayable. The operator may then choose to scale the original data by a variety of methods including logarithmic, straight average, or parabolic average. The program accomplishes the summation in real time with the use of a state table (named AVERAGE) and the signal processor board of the image processor. 
SLOWAVG performs basically the same functions as FASTAVG, but the data is sumned at a slower rate. The minimum delay between successive grabbed frames is 1 second. SLOWAVG also uses a state table (named SAVG).

Programs ANALYZE and ANALFILE are not true image processing programs since they do not actually result in a new or altered image. The purpose of ANALYZE is to convert the greyscale values provided by the image processor and QLIS for a given image into quantitative radiance numbers. The frame to be operated upon may be the currently displayed frame, a grabbed frame, or a frame already in storage. The selected frame is first corrected to remove a radial gain gradient present in the system. Then the program allows the user to choose a single point or circular area of interest for analysis. The output of the program consists of the average greyscale of the area of interest and the corresponding radiance. As an option, the user may specify a listing of the greyscale and radiance of each individual pixel within the area of interest.

Since the QLIS imaging system is not a dispersing instrument (that is, it neither provides nor understands color information), the system spectral response, sample spectral content, and calibration source spectral content must be known in order to calculate a valid radiance. These values are input to the computer with program ANALFILE. The ANALFILE program stores files under operator specified names, making the data available to ANALYZE. While running the ANALYZE program, the operator is asked for the file names of the various spectral curves and system gain response. For further information on how this data is used in calculating the radiance, see the section of this manual entitled CALIBRATION AND RADIOMETRIC CALCULATIONS.

The calibration of the QLIS is carried out by program CALIBRAT. Further details of the operation of CALIBRAT are also given under CALIBRATION AND RADIOMETRIC CALCULATIONS.

\section{IMAGE PROCESSOR STARTUP}

The image processor is automatically powered up when the Microvax II host computer is switched on. The image processing command file, programs, drivers, and files are stored on one of the internal disks, under the SYS\$SYSROOT: [VIOS] directory. As mentioned in the VIOS software manual, the 
command file which loads the VIOS driver must be executed before the image processing boards can be used. The command file is named "QVLOAD.COM" and must be executed using "@QVLOAD.COM, <Return>" each time the system is powered up. The command file could also be executed by the site-specific start-up command file SYSTARTUP.COM. Such a configuration would automatically load the VIOS driver every time the Microvax II is booted. 


\section{CALIBRATION AND RADIOMETRIC CALCULATIONS}

This section describes the procedure for calibrating the QLIS. The calculational method for greyscale to radiance conversion is also described.

\section{CALIBRATION PROCEDURE}

The calibration should be done, at least initially, whenever the system is powered up to be used for quantitative work. If experience with the calibration indicates that it need only be done at greater intervals, the experimentalist is certainly free to do so.

The actual procedure for performing the calibration is quite straightforward:

1) Power up the computer and imaging system and allow them to warm up for a reasonable amount of time such as 30 minutes. During this time, be careful to avoid exposing the image intensifier to bright light as such exposure may cause the noise level to increase and stay above normal for some time. In addition, the calibration source should be kept in a dark place, such as cabinet drawer, with the sliding shutter on the source holder closed. If the calibration source phosphor is exposed to room light, it will glow much more brightly than it should for a period of time. If this accidently happens, the source must be placed in a dark place for at least one hour to allow the phosphor to return to its proper activity.

2) Slip the calibration source over the end of the nylon coupler at the input to the fiber optic image transfer system. It should slip on easily, the slots in the calibration source mating with the three set screws on the nylon coupler. If the source dos not slip on easily, do not force it! Examine the nylon coupler and calibration source for alignment problems, etc., if necessary.

3) Darken the room and use the CALIBRAT program to carry out the calibration. The program gives instructions and prompts for user input as necessary. When execution of CALIBRAT is complete, the calibration grey scale and gain are stored in file CALDATA.DAT; $n$ which is later accessed by ANALYZE. 
4) Return the gain to zero. Remove the calibration source. As usual, check the monitor to see that the imaging system is not being over-exposed. If the display is flashing, immediately switch off the power supply to the image intensifier. Subdue the lighting conditions before turning the image intensifier back on.

\section{RADIANCE CALCULATION}

The radiance calculation for the QLIS is relatively straightforward. The radiance values are referenced to a self-luminous carbon-14 activated calibration source. The source is highly stable owing to the 5730 year halflife of the $\mathrm{C}-14$ activator. Further detail on this source has been provided in the sections of this manual entitled HARDWARE DESCRIPTION Calibration Source and CALIBRATION AND RADIOMETRIC CALCULATIONS Calibration Procedure.

In general terms, the radiance calculation is based on comparing the QLIS grey value for the calibration source with that of the test sample. Since the radiance of the calibration source is known, the radiance of the sample is found by proportions. The process of determining the radiance of the sample is begun with program CALIBRAT. As described elsewhere in this manual, the calibration source is placed at the object plane of the QLIS fiber optic input. The resulting grey value of the image $\left(v_{c}\right)$ and the intensifier gain setting $\left(g_{c}\right)$ are stored by CALIBRAT in a file in the host computer. When an acquired image of the test sample is to be analyzed, an area of interest is selected by the user. The average grey value of that portion of the image $\left(v_{s}\right)$ is found and the intensifier gain setting $\left(g_{s}\right)$ is entered by the user.

Since the image processor is limited to 256 grey scale divisions, a wide range of input radiances can result in an identical grey value due to the variable gain of the intensifier. Therefore, the relation between grey scale, gain setting, and relative image brightness must be known. A typical example of the relationship between these three variables is shown graphically in Figure 10. Notice that the abscissa is given in neutral density values and that the gain curves are spaced at intervals of 100 . This arises due to the experimental method used in determining the relationships wherein a source of sufficient radiance to give a grey value of 256 at zero gain is successively 
TYPICAL GREY VALUE. GAIN, SCALE FACTOR RELATIONSHIP GAIN VARIED FROM TO 1 DEV IN STEPS OF 1 घE

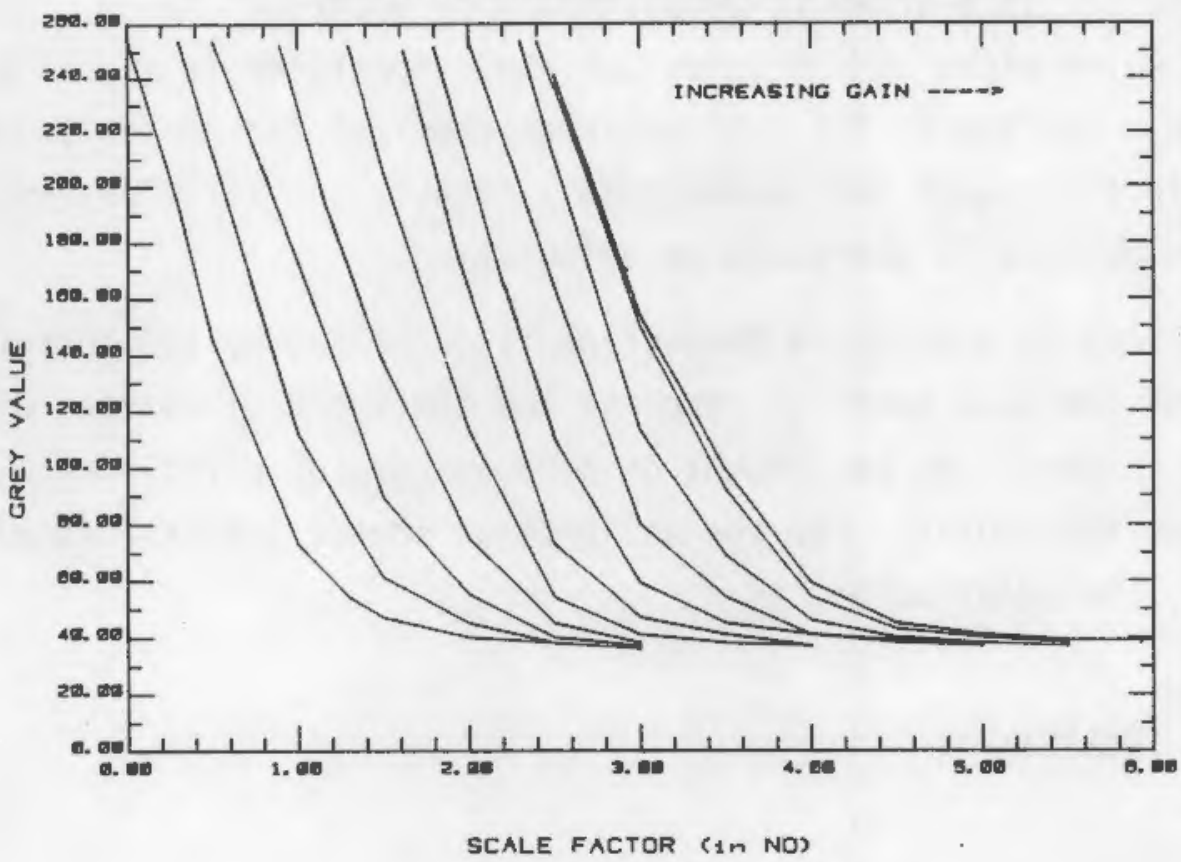

FIGURE 10. Graphical Representation of Relationship Between Grey Value, Intensifier Gain, and Scale Factor

reduced via neutral density filters to give lower and lower radiances as the intensifier gain is increased in steps of 100 . To convert from neutral density values to the scale factor, consider that the scale factor equals 10-neutral density.

The QLIS analysis program uses the calibration source and test sample grey values and gain settings, along with a table of data such as that in Figure 10, to interpolate a scale factor for each input. The scale factors are a function of grey value $(v)$ and gain setting $(g)$, so they may be notated as $s\left(v_{c}, g_{c}\right)$ and $s\left(v_{s}, g_{s}\right)$ for the calibration and test sample scale factors, respectively. The grey values for the calibration source and test sample are multiplied by their respective scale factors, giving an effective grey value for each that is independent of the intensifier gains used. Since the radiance $\left(L_{c}\right)$ of the calibration source is known, the radiance of the test sample can subsequently be found by proportion.

There is another consideration, however, in that the QLIS is a spectrally integrating instrument. That is, the spectral content of the calibration 
source and the test sample is lost at the image intensifier stage; the output of the intensifier is a green ( $P-20$ phosphor) "monochrome" image. In addition, the newvicon which picks up the output of the intensifier is itself a monochrome device. Therefore, the QLIS converts spectral (or "color") input information to grey scale (or "monochrome") output, and this conversion must be taken into account in the radiance calculation.

The spectral to grey scale conversion is described by the integral of the product of the QLIS spectral response and the spectral content of the input. This integral can be thought of as a conversion efficiency $\eta$. Figure 11 illustrates this point. For the calibration source and test sample, respectively, the relationship is

$$
\eta_{C}=\int_{\lambda_{1}}^{\lambda_{2}} Q(\lambda) C(\lambda) d \lambda
$$

and

$$
\eta_{S}=\int_{\lambda_{1}}^{\lambda_{2}} Q(\lambda) S(\lambda) d \lambda
$$

where $Q(\lambda)$ is the spectral response of the QLIS, $C(\lambda)$ and $S(\lambda)$ are the spectral content of the calibration source and test sample, respectively, and $\lambda$ is wavelength. In the QLIS analysis program, the limits of integration ( $\lambda_{1}$ and $\left.\lambda_{2}\right)$ are taken to be 400 and $700 \mathrm{~nm}$ since the calibration source and test sample spectral contents are zero beyond these limits. The conversion efficiencies for the calibration source and test sample are divided into their respective grey values so that the radiance calculation accounts for the differing spectral contents of the samples.

All of the factors for the radiance calculation have now been discussed, so the equation for radiance $(L)$ can be assembled:

$$
L=\frac{v_{s}}{v_{c}} \cdot \frac{s\left(v_{s}, g_{s}\right)}{s\left(v_{c}, g_{c}\right)} \cdot \frac{\eta_{c}}{\eta_{s}} \cdot L_{c}
$$




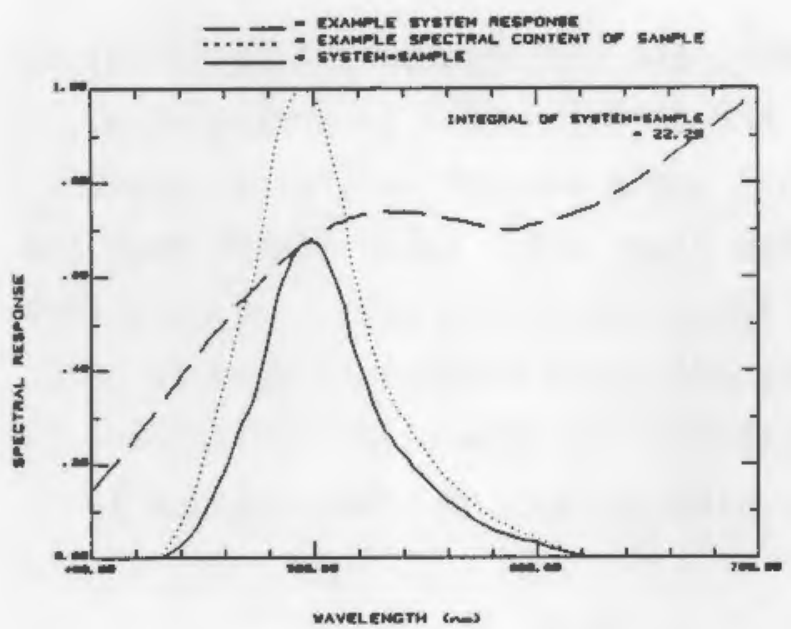

a) Low Efficiency

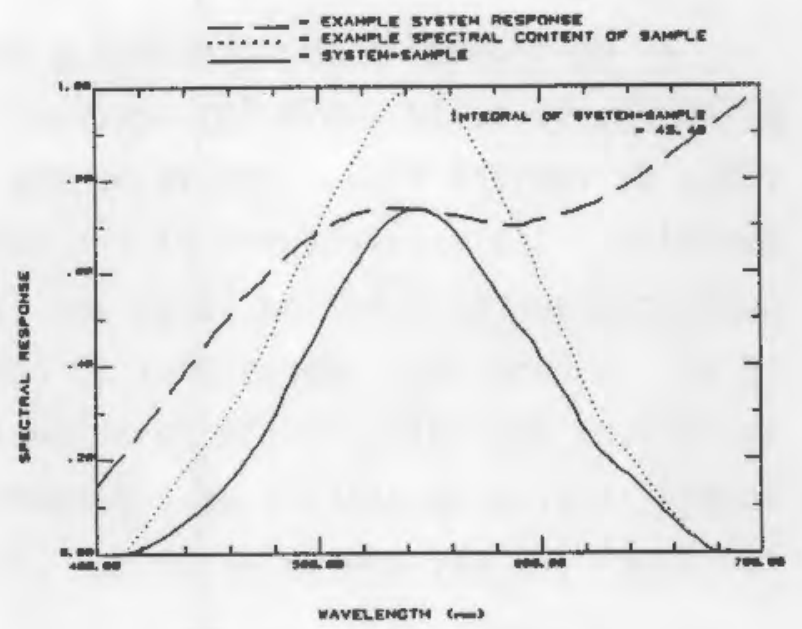

b) High Efficiency

FIGURE 11. Illustration of Spectral-to-Grey Scale Conversion Efficiency

All of the variables on the right side of the radiance equation are dimensionless except for $L_{c}$, the calibration source radiance, which has units of watts $/ \mathrm{cm}^{2}-\mathrm{sr}$ as expected.

\section{RADIANT FLUX CALCULATION}

The radiance $L$ is converted to a radiant flux ( $P$, also called power, in units of watts) by multiplying by the appropriate area in $\mathrm{cm}^{2}$ and solid angle in steradians. The area contained in the region of interest of an image selected by the user is directly related to the number of pixels in that region. Therefore, if the area per pixel (A) is known, it is a simple matter to count up the number of pixels $(n)$ and multiply by the area per pixel to get the area of the region. The area per pixel can be found by placing a circular mask over the fiber optic image pipe input. A convenient mask is a piece of black paper from which a disk of known area is cut out. With some low-level illumination, the QLIS can be directed to grab a frame of this diskshaped image. Subsequently, one of the programs such as ANALYZE can be used to select a region of interest which corresponds exactly to the perimeter of the image of the disk. When this region of interest is selected, the number of pixels contained in it is reported by the computer along with the average grey scale of the region. Since the corresponding area is known, the area per pixel can now be found and entered in the ANALYZE program code as a parameter. 
As mentioned in the previous paragraph, the radiance must also be multiplied by the solid angle $(\Omega)$ accepted by the QLIS in order to arrive at a value of radiant flux. The value for solid angle was not determined experimentally. The manufacturer of the coherent fiber optic taper states that the numerical aperture of the large end (the input end in the QLIS configuration) is .5. A numerical aperture of .5 corresponds to an acceptance cone of .842 steradians according to the relation illustrated in Figure 12 . This value is stored in program ANALYZE as a parameter. The conversion from radiance to radiance flux may now be written

$$
P=L_{C} A n \Omega
$$

When the radiant flux for each individual pixel is desired, the analysis program calculates the values in the same manner as for the complete area of

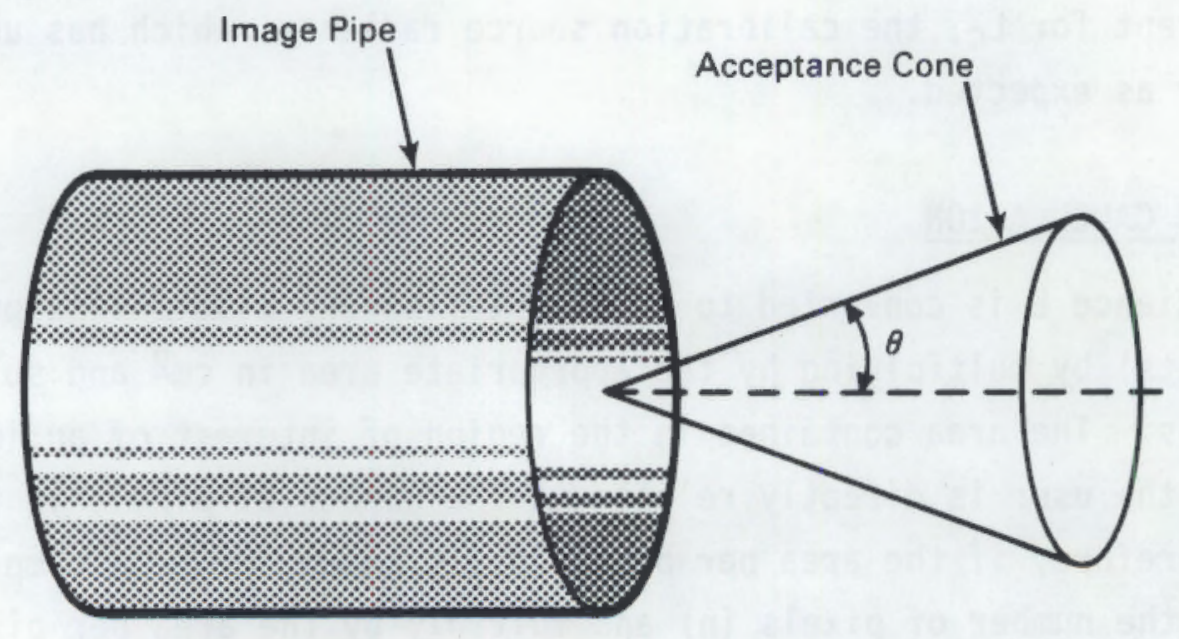

$\operatorname{Sin} \theta=$ N.A.

$$
\begin{aligned}
& \theta=\operatorname{Sin}^{-1} \text { N.A. } \\
& \Omega=4 \pi \operatorname{Sin}^{2}\left[\frac{1}{2}\left(\operatorname{Sin}^{-1} \text { N.A.) }\right]\right.
\end{aligned}
$$

FIGURE 12. Relationship Between Numerical Aperture and Solid Angle 
interest. In this case, however, the grey value of each pixel is individually considered instead of the average grey value of all of the pixels in the area of interest. As the values are calculated, the pixels' $X, Y$ coordinates and corresponding radiant fluxes are listed to the host computer's output device.

\section{PHOTONS PER SECOND CALCULATION}

The QLIS analysis program also provides a listing of the photons per second generated by the sample under test. The calculation begins with an integration of the sample spectral content. Following this, the spectrum is divided into discrete bands 10 nanometers wide. The integral of the first band is found so that the fraction $(F)$ of the total spectrum contained in the band may be calculated and hence the radiant flux contributed by the band. Note that the total radiant flux $P$ has already been calculated by the analys is program. Next, the energy of a photon with the wavelength lying in the center of the band is calculated in eV per photon and subsequently converted to joules per photon. Since $1 J=1 W-s$, this energy per photon $\left(E_{p}\right)$ can be expressed in watt-seconds per photon. Thus, the number of photons per second $\left(N_{p h}(\lambda)\right)$ in the band is found by

$$
N_{p h}(\lambda)=\frac{F \cdot p}{E_{p}}
$$

The procedure is repeated for each band. The QLIS analysis program reports each wavelength band and its corresponding value of $\mathrm{N}_{\mathrm{ph}}$. In addition, the program reports the total number of photons per second, which is just a sum of the individual band contributions. 


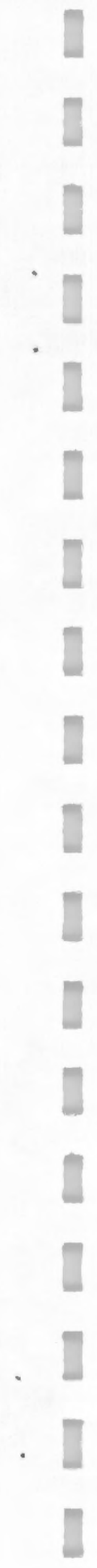


APPENDIX A

SYSTEM ENHANCEMENTS 


\section{APPENDIX A}

\section{SYSTEM ENHANCEMENTS}

Several system enhancements have been provided in the period between delivery of the QLIS and completion of this document. The enhancements provide increased functionality and ease of use. These items include a high-level software graphics library, computer-controllable power supply, RS-232 card for the host computer, a professional quality VHS video tape recorder, and a chrominance encoder.

The graphics software is entitled DISSPLA. It provides great versatility in display of data, results, etc. A few of the many features include twodimensional and three-dimensional plotting, contouring, multiple fonts, definable axes, and surfacing. All available options were purchased, including CODEBOOK, a software routine which enables customized graphics routines to be quickly assembled from pre-written sample programs. CODEBOOK should be particularly useful in learning to use the DISSPLA library.

The computer controllable image intensifier power supply is the OPELCO \#1390ps. It functions in the same manner as the manual setting power supply, but provides an RS-232 controllable capability. In remote control mode, the power supply will free the user from the need to pause and turn the gain setting knob of the supply. When the necessary commands are inserted into the QLIS software, the intensifier gain can be automatically reduced or increased as needed.

In order to use the remote control power supply, it is necessary that the host computer have an RS-232 port available for dedication to the power supply. This requirement is satisfied by the DEC DZQ1I-M, a four line asynchronous multiplexed RS-232 card. The card is designed to be plugged directly into the host computer backplane.

A professional-quality VHS video recorder is added to the system to provide for easy and inexpensive real-time data storage. The stored video data can be fed to the image processor for analysis at the user's convenience. The recorder, a Panasonic AG-6300, provides high-level capabilities such as multiple speed forward and reverse, dial search, repeat loop, and real-time tape counter. 
The LENCO \#CCE-850 chrominance encoder allows conversion of the threesignal RGB output of the image processor to standard NTSC composite video. With the encoder, the output of the image processor, whether black and white or color, can be accurately stored on video tape. Without the encoder, only the green output of the RGB signal could be recorded since it alone carries the video sync information. 


\section{APPENDIX B}

COMPONENT VENDOR LIST 


\section{COMPONENT VENDOR LIST}

Image intensifier system

Model \#KS-1380G image intensifier

Mode I \#ITC-510 newvicon camera

OPELCO, InC.

P.0. Box 17127

Washington D.C. 20041

Contact: Herman Esser

703-471-0080

Fiber optic image transfer

Model (none - custom item)

INCOM, Inc.

205 Chapin Street

Southbridge MA 01550

Contact: Jerry Burke

617-765-9151

Image processor

Mode1 \#QVG/AFA/SP-423-08-83

Datacube, Inc.

4 Dearborn Rd.

Peabody MA 01960

Contact: Glen Ahearn

617-535-6644

Image processor driver

Model \#VIOS-VMS-TK50

Process Software Corporation

35 Montague Road

Amherst MA 01004

Contact: Phil Denzer

413-549-6994 


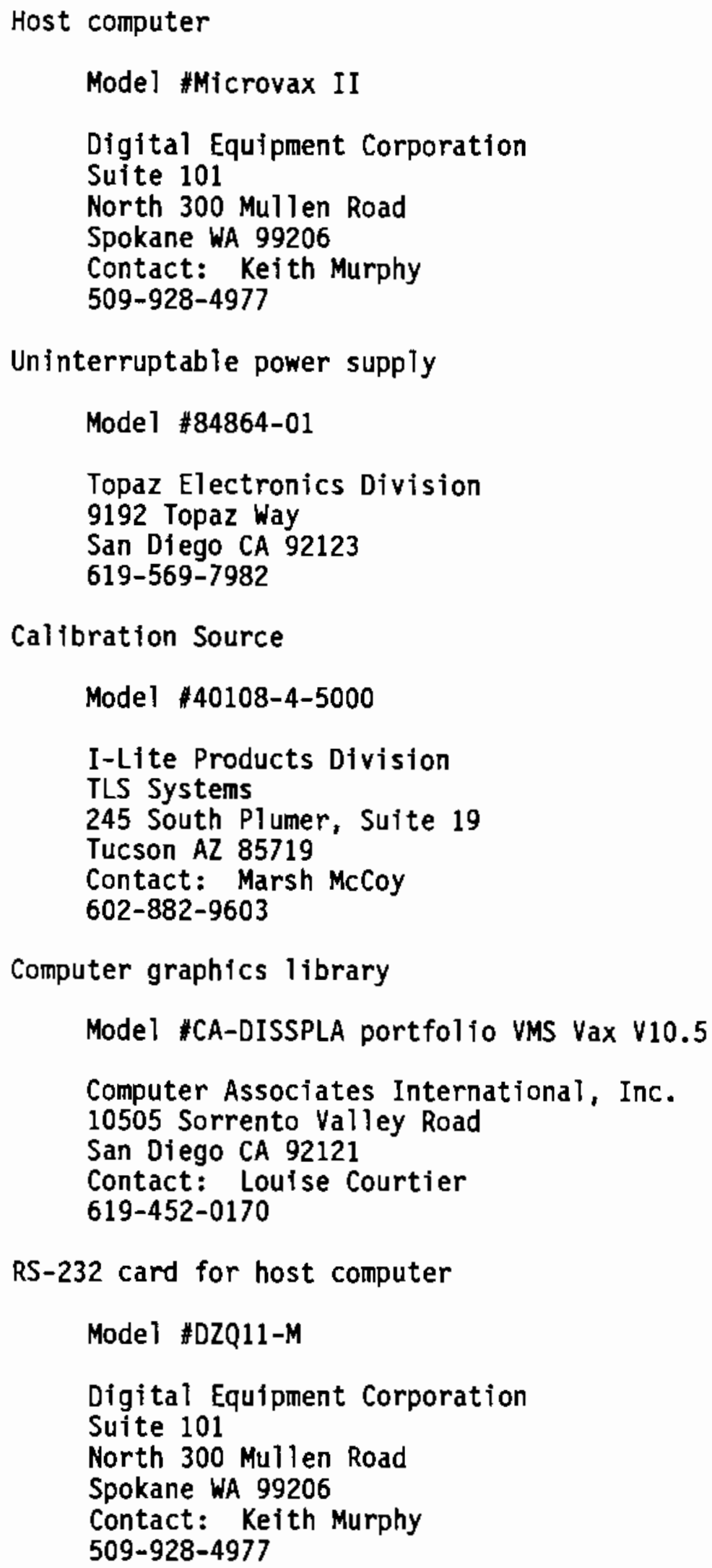

RS-232 card for host computer

Model \#DZQ11-M

Digital Equipment Corporation

Suite 101

North 300 Mullen Road

Spokane WA 99206

Contact: Keith Murphy

509-928-4977 
Remote control image intensifier power supply

Model \#1390PS

OPELCO, InC.

P.0. Box 17217

Washington D.C. 20041

Contact: Herman Esser

703-471-0080

VHS recorder

Model \#Panasonic AG-6300M0

Northwest Electronics, Inc.

P.0. Box 3047

Spokane WA 99220

Contact: Lynn Treffry

800-46B-9274

Chrominance encoder

Model \#CCE-850

LENCO

P.0. Box 348

Jackson MO 63755

Contact: Customer service

314-243-3147 
APPENDIX C

MECHANICAL DRAWINGS 


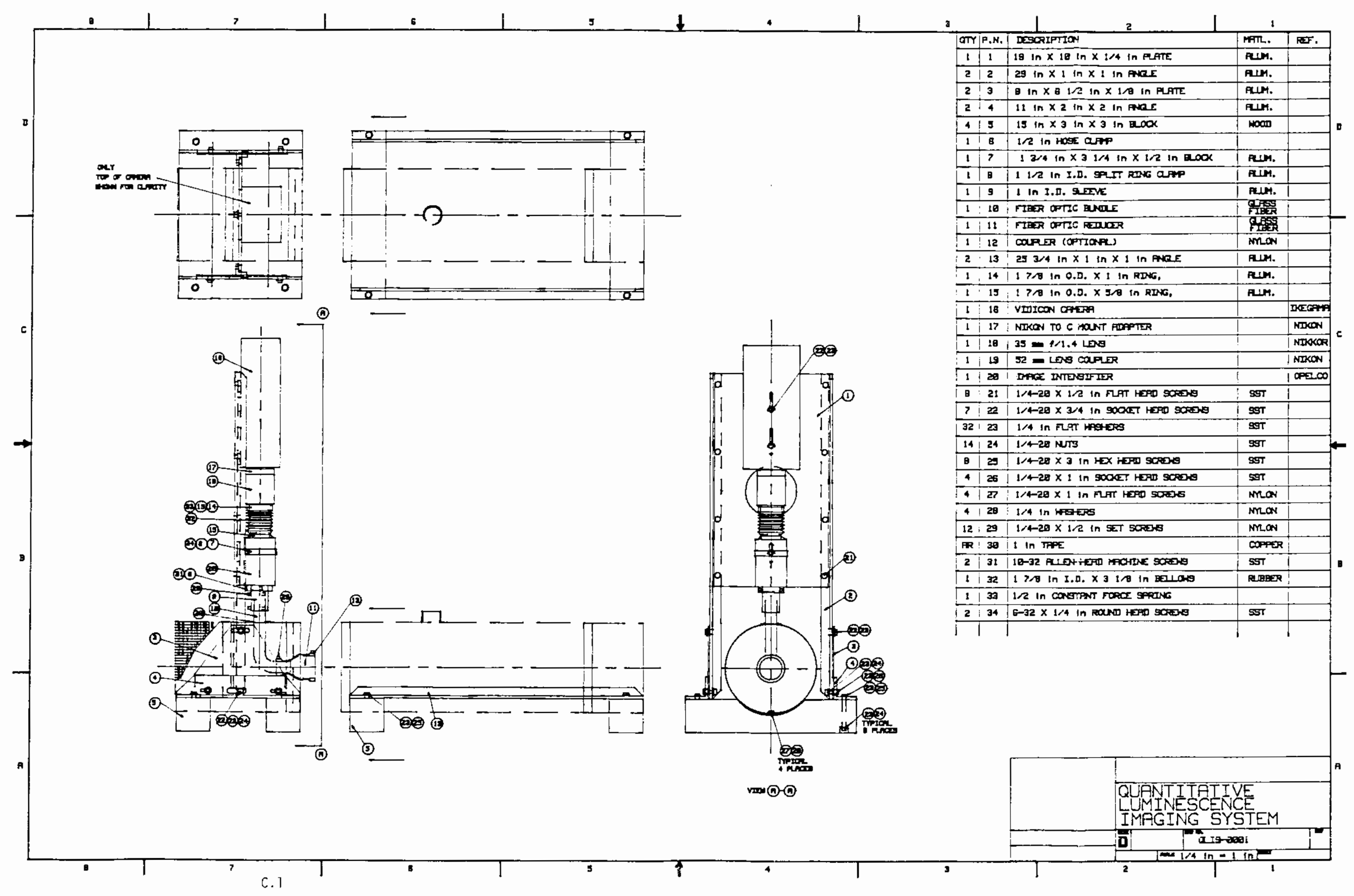




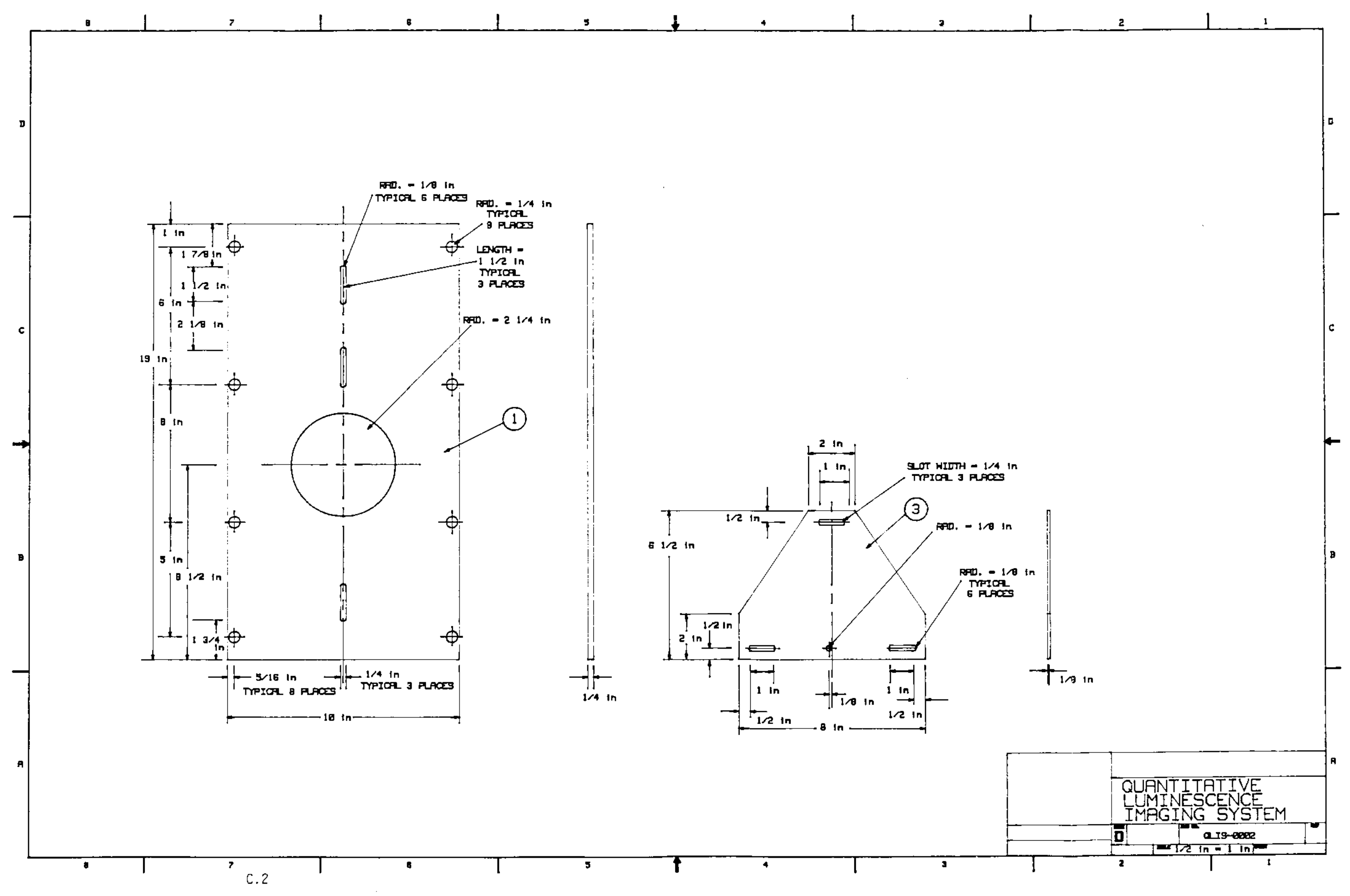




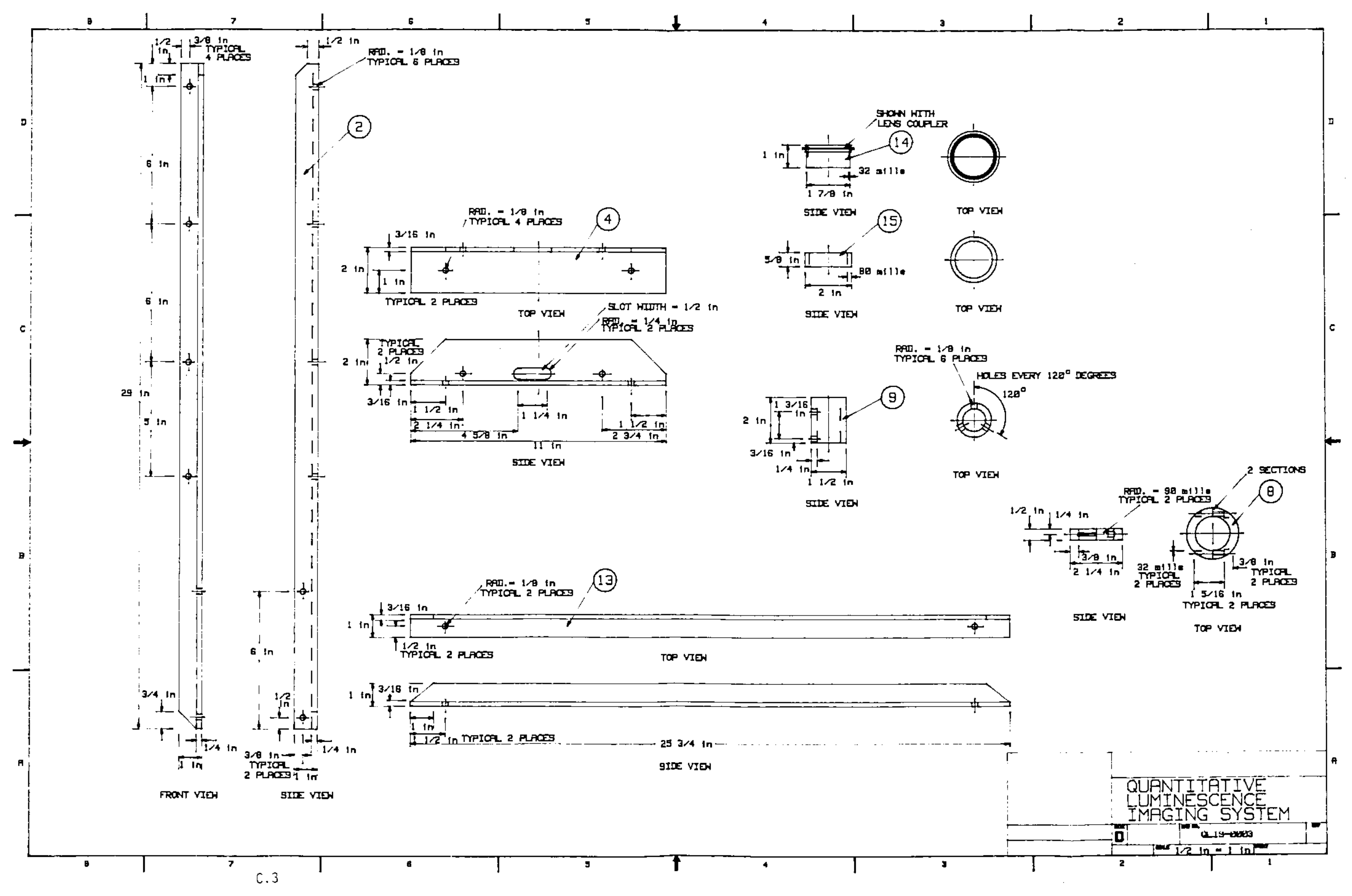




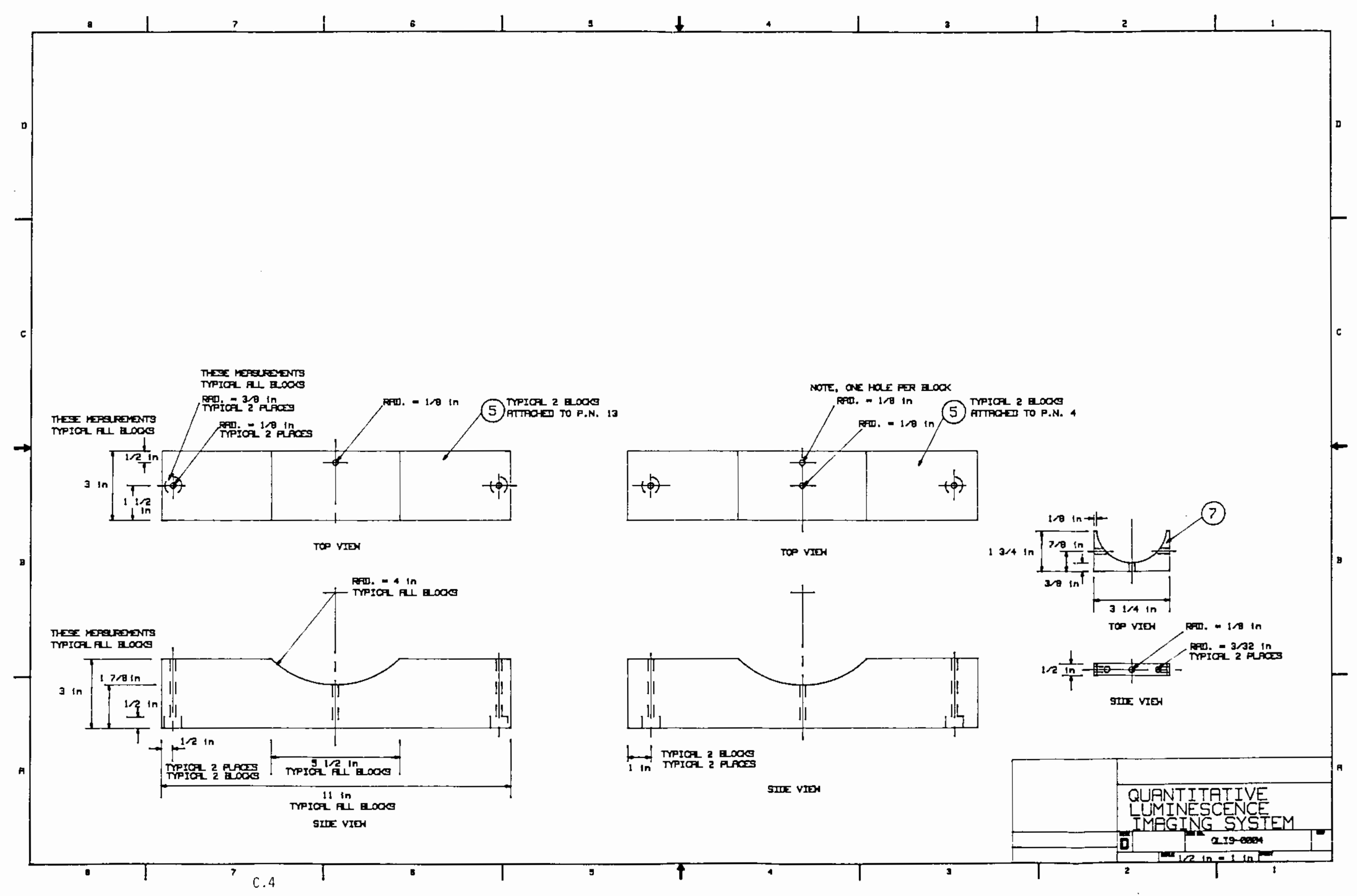




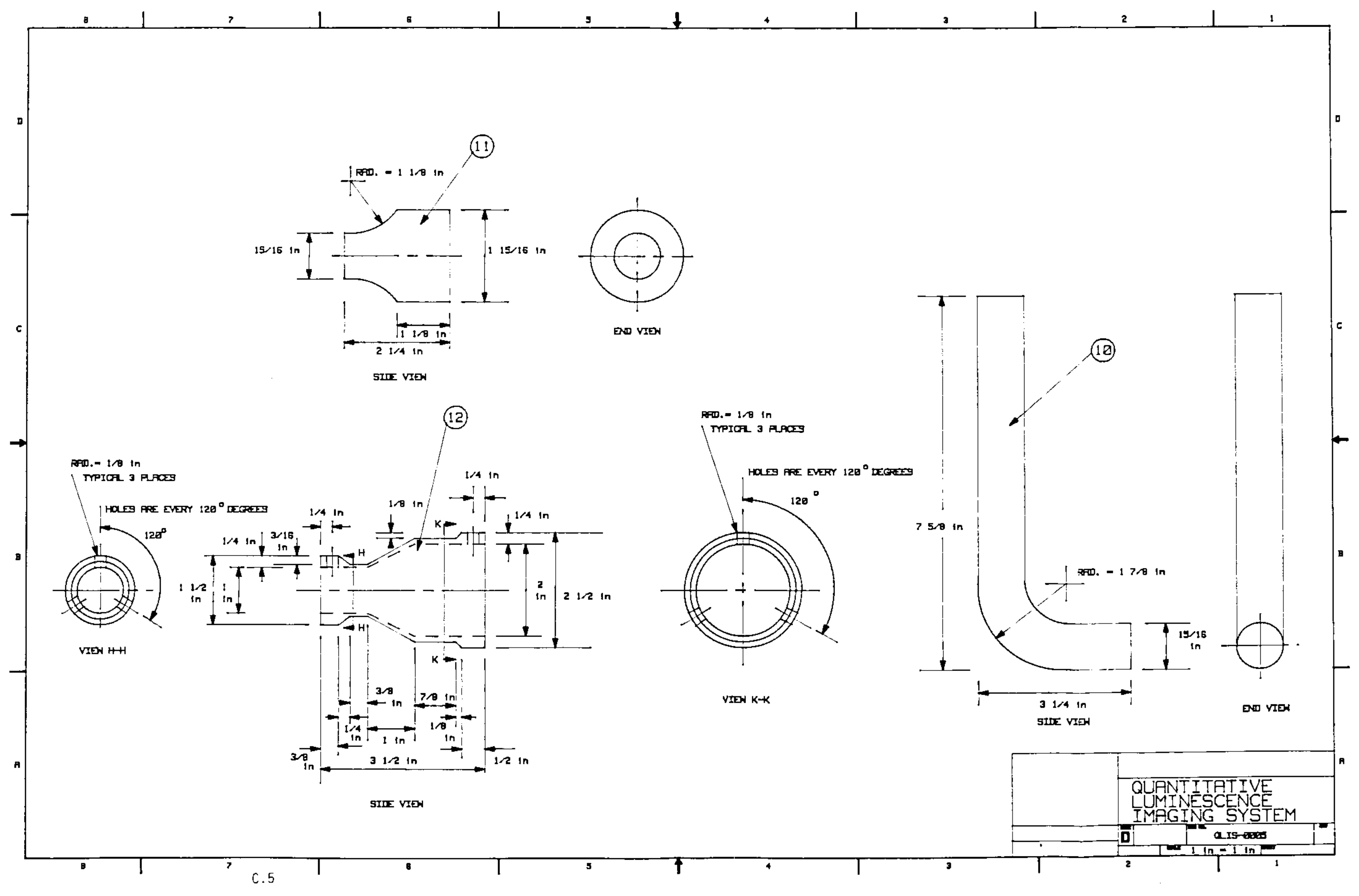




\section{DISTRIBUTION}

No. of

Copies

\section{OFFSITE}

2 J. L. Kiel

USAF School of Aerospace Medicine

Brooks Air Force Base TX 78235

2 D. N. Erwin

USAF School of Aerospace Medicine

Brooks Air Force Base TX 78235

J. C. Mitchell

USAF School of Aerospace Medicine

Brooks Air Force Base TX 78235

D. M. Simmons

USAF School of Aerospace Medicine

Brooks Air Force Base TX 78235

2 DOE/Office of Scientific and Technical Information

\section{ONSITE}

DOE Richland Operations Office

J. J. Sutey

10 Pacific Northwest Laboratory

C. R. Batishko

K. A. Stahl

Patent office

Publishing Coordination (2)

Technical Report Files (5) 
